Review Article

\title{
Pushing CT and MR Imaging to the Molecular Level for Studying the "Omics": Current Challenges and Advancements
}

\author{
Hsuan-Ming Huang and Yi-Yu Shih \\ Healthcare Sector, Siemens Limited Taiwan, 2 F No. 3, Yuan Qu Street, Nan Gang District, Taipei 11503, Taiwan \\ Correspondence should be addressed to Yi-Yu Shih; yiyushih@gmail.com
}

Received 18 October 2013; Revised 26 December 2013; Accepted 24 January 2014; Published 13 March 2014

Academic Editor: Yu-Hua Dean Fang

Copyright (c) 2014 H.-M. Huang and Y.-Y. Shih. This is an open access article distributed under the Creative Commons Attribution License, which permits unrestricted use, distribution, and reproduction in any medium, provided the original work is properly cited.

During the past decade, medical imaging has made the transition from anatomical imaging to functional and even molecular imaging. Such transition provides a great opportunity to begin the integration of imaging data and various levels of biological data. In particular, the integration of imaging data and multiomics data such as genomics, metabolomics, proteomics, and pharmacogenomics may open new avenues for predictive, preventive, and personalized medicine. However, to promote imagingomics integration, the practical challenge of imaging techniques should be addressed. In this paper, we describe key challenges in two imaging techniques: computed tomography (CT) and magnetic resonance imaging (MRI) and then review existing technological advancements. Despite the fact that CT and MRI have different principles of image formation, both imaging techniques can provide high-resolution anatomical images while playing a more and more important role in providing molecular information. Such imaging techniques that enable single modality to image both the detailed anatomy and function of tissues and organs of the body will be beneficial in the imaging-omics field.

\section{Transition from Morphological to Molecular Imaging}

Over the past few decades, imaging data have been widely used to study how body system work together at the organtissue level. In contrast, omics data obtained from genomics, metabolomics, proteomics, and pharmacogenomics have been used to provide biological information at the cellular and molecular level. Such omics data may provide a great opportunity to understand the development and progression of diseases. With the integration of imaging and omics data, scientists may find new insights into the mechanism of disease and its treatment. To integrate images as part of the omics, imaging techniques are needed to be safe, fast, accurate and convenient. More importantly, these techniques should have the ability to provide anatomical and functional information.

Among several imaging techniques, computed tomography (CT) and magnetic resonance imaging (MRI) have the potential to satisfy the above requirements. First, human anatomical structures can be imaged using both CT and MRI.
Second, recent studies showed that genomics data were correlated with functional (molecular) CT and MRI data [1-8]. It is noteworthy that MR spectroscopy is a conventional technology which could approach in vivo molecular and metabolism information, and there are many valuable results combined with various omics data in different clinical applications, such as oncology and neural diseases, in recent decades [9-12]. Moreover, several nanoparticles such as gold nanoparticles $[13,14]$ and polymer-coated bismuth sulfide nanoparticles [15] have emerged as novel CT contrast agents for cancer detection. Similarly, some novel genetically encoded MRI contrast agents and advanced sequence technologies have been used to detect gene expression [16-19]. Finally, due to the advantages of cost, convenience (for CT), and safety (for MRI), both CT and MRI will be appropriate imaging techniques for imaging-omics integration.

To build and validate the relationship between omics data and imaging features, there are several limitations that need to be considered. In CT, both anatomical and functional data can be obtained within a few minutes. However, multiple CT scans are required, and patients would receive a high dose 


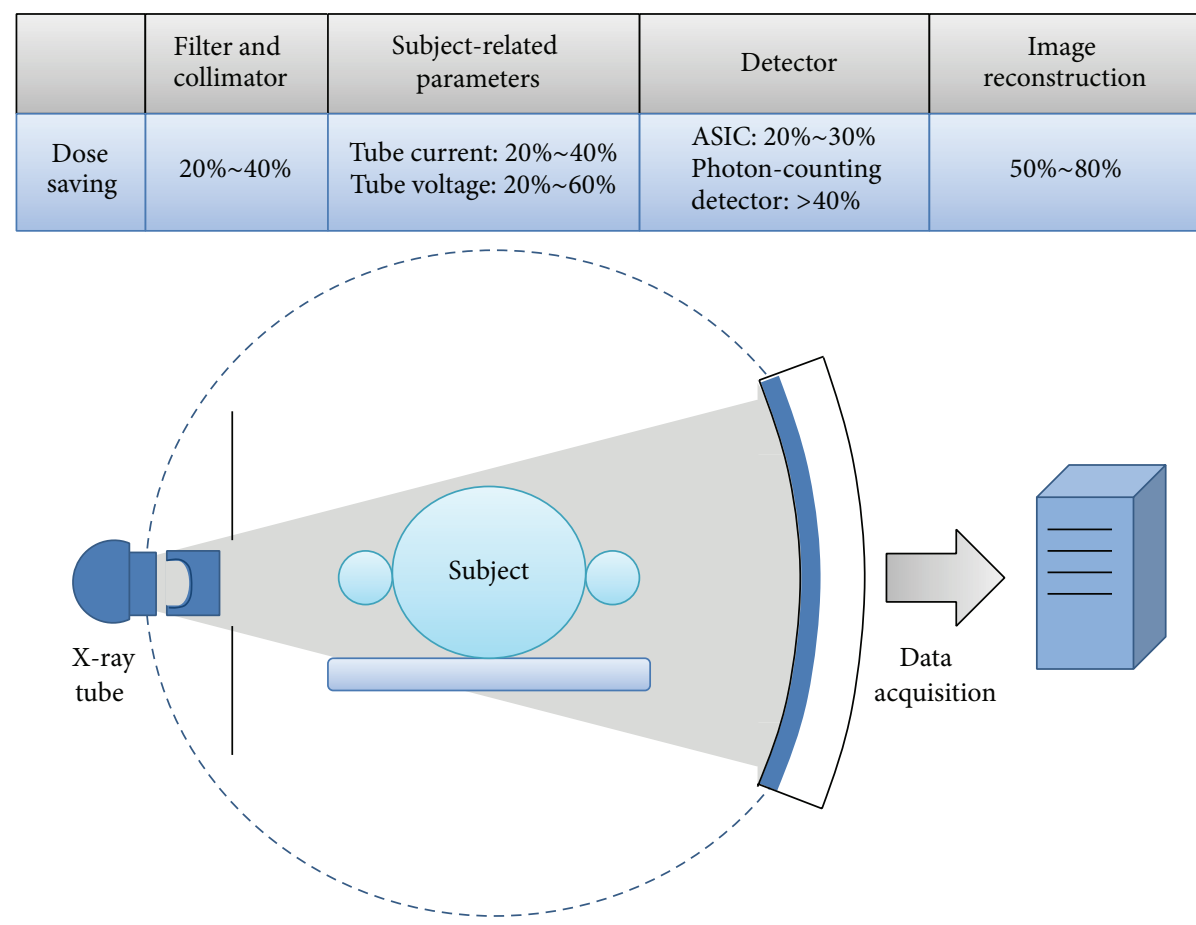

FIgURE 1: The key component of a CT system and the dose-saving potential of each component.

of radiation that may increase the risk of developing cancer. As a result, dose reduction in $\mathrm{CT}$ is an important issue for the integration between CT and omics data. Unlike CT, MRI does not use ionizing radiation. However, it takes a relatively long acquisition time to obtain anatomical and functional data and may not be acceptable for uncooperative subjects. Thus, acceleration is an important issue for the integration between MRI and omics data. Since the radiation dose of CT and acquisition time of MRI can determine the quantity and quality of anatomical and functional data, they are the key to imaging-omics integration. Hence, the aim of this paper is to review existing technological strategies and new directions that are used for reducing radiation dose in CT and acquisition time in MRI.

\section{Dose Reduction in Computer Tomography}

Nowadays, CT is one of the most commonly used imaging modalities because CT scanning is simple, fast, noninvasive, and accurate. Despite the tremendous benefits of CT scans, radiation dose from CT scans may be a potential risk factor for cancer development. In order to keep radiation as low as reasonably achievable (ALARA), techniques and strategies for reducing patient dose are highly desirable. We would discuss the dose-saving strategies of each component in a CT system shown in Figure 1.

\subsection{CT Data Acquisition and Processing}

2.1.1. Beam Filtration. Generally, X-ray beam emitted from an $\mathrm{X}$-ray tube contains a spectrum of photon energies. Among these X-rays, soft (i.e., low-energy) X-rays are not hard enough to efficiently penetrate the subject. As a result, soft Xrays contribute to patient radiation dose without providing useful information. In order to reduce the radiation dose to a patient, X-ray filters that block soft X-rays were developed. Due to differences in attenuations between the central and peripheral regions, commercial CT scanners are commonly equipped with a beam-shaping filter or bow-tie filter instead of the flat filter $[22,23]$. In addition, specific beam-shaping filters that adapt to different examinations such as head, body, and cardiac were developed [24]. One possibility for further reducing patient dose is to use dynamic bow-tie filters which modify X-ray beam as a function of view angle $[25,26]$.

2.1.2. Collimator. Since the focal spot in the X-ray tube is not truly a point source, beam coverage in some regions is partially irradiated. These regions are called penumbra. In multislice CT, overbeaming caused by focal spot penumbra does not contribute to image reformation. As a result, exposure to such radiation is unnecessary. In multislice row CT, thick beam collimation has better radiation utilization [27]. This is because radiation dose due to overbeaming constitutes a smaller percentage of total absorbed doses. However, thick collimation only allows reconstruction of thick sections. To reconstruct thin sections, thin collimator that reduces radiation utilization is required. This indicates that beam collimation should be selected based on the requirements of clinical examinations.

In multislice CT, spiral scan is the commonly used scanning mode which results in overscanning or overradiation $[28,29]$. To obtain sufficient data for reconstructing image at the beginning and end of the $z$-axis scan range, at least an additional one-half of a gantry rotation is prerequisite. This 
so-called overscanning contributes to patient dose but does not contribute to image reformation. Therefore, a hardwarebased solution that uses the dynamic or adaptive collimator to block the pre- and postspiral radiation was introduced and used in clinical practice [30,31].

2.1.3. Detector. In a CT system, X-ray detector is probably the most important component that converts incident X-ray beam into digital signals. In the 1980s, xenon-gas ionization chamber detectors were commonly used in CT. However, with improvements in technology, xenon-gas detectors are replaced by solid-state detectors (e.g., gadolinium oxysulfide) which have higher quantum efficiency. In normal-dose CT examinations, noise from the electronic components of the detector system is negligible and has no significant impact on image quality. However, in low-dose CT examinations, electronic noise becomes one of the most significant factors determining image quality. Hence, it is important to figure out how electronic noise in low-dose scans can be reduced. In recent years, two new CT detectors, GE's Gemstone Detector and Siemens' Stellar Detector, were introduced into the market. Gemstone Detector made from a garnet-based scintillator enables improvements in spatial resolution and signalto-noise ratio (SNR). Besides, Stellar Detector that combines the photodiode and the analog-to-digital converters (ADC) in one application specific integrated circuit (ASIC) allows a $\sim 20 \%$ reduction in electronic noise. As a result, CT scanners equipped with new detectors are expected to have a reduction in patient dose.

Generally, X-ray detectors currently used in clinical CT scanners are energy integrating. In contrast to conventional energy-integrating detectors, photon-counting detectors not only count each photon individually but also record the energy level of each detected photon. Based on the energyresolving information, photon-counting detectors have the ability to eliminate electronic noise as well as scattered radiation [32,33]. In addition, photon-counting detectors with energy discrimination capabilities can be used to improve SNR by assigning an energy-dependent weighting factor to each individual photon [34-36]. Due to the improvement of SNR, the patient dose can be further reduced while maintaining acceptable image quality.

In practice, photon-counting detectors are not widely used in CT systems. This is primarily because the fluxes of incident photons are too high. Under high flux irradiation, the pulse resolving time is relatively too long to allow the detector recording each individual photon. As a result, overlapping pulses which lead to a spectral distortion and a loss of counts may occur. This phenomenon is called pulse pileup [37]. Although a clinical CT scanner equipped with photoncounting detectors is not available yet, recent advances in detector electronics and semiconductor detector materials have been contributed to developing a photon countingbased micro-CT system [38-40]. We expect that a photon counting-based CT will soon be ready for clinical study.

2.1.4. Tube Current. Among many scanning parameters, tube current is one of the important factors determining the radiation dose delivered to the patient. Hence, one commonly used technique to reduce radiation exposure is to adapt the tube current according to the patient's size, shape, and attenuation. Methods that automatically modulate the tube current to adapt differences in patient attenuation are known generically as automatic exposure control (AEC) [41-44]. Now, in most modern CT scanners, AEC can perform automatic adjustment of tube current both during tube rotation (i.e., angular modulation) and along the $z$-axis of the patient (i.e., $z$-axis modulation). In angular modulation, the tube current is adjusted to adapt differences in attenuation between anteroposterior and lateral positions. Unlike angular modulation, the $z$-axis modulation adjusts the tube current to accommodate differences in attenuation along the $z$-axis of the patient (e.g., shoulder and abdomen). The main aim of AEC is not only to modulate tube current but also to deliver the right dose in order to achieve adequate image quality for each individual patient and each diagnostic task. The underlying principle of AEC is similar; however, due to different definitions of image quality, AEC systems from four major CT manufactures are implemented somewhat differently [45]. Despite these different implementations, the AEC techniques used in modern CT scanners contribute to a significant reduction in radiation dose to the patient while maintaining the adequate image quality [45].

2.1.5. Tube Potential. To date, lowering tube potential has been demonstrated to improve image quality or reduce radiation dose in several clinical CT exams [46-52]. The underlying principle is based on the fact that iodine has an increased attenuation (i.e., higher contrast) as photon energy decreases toward the $k$-edge energy of iodine. This indicates that for the same radiation dose, the lower tube potential can yield better image quality than the higher tube potential. Alternatively, the improvement in image quality can contribute to reducing patient dose. However, with the increase of the patient size, low kilovoltage $(\mathrm{kV})$ settings would lead to an increase in image noise. Hence, dose reduction using lower tube potential is feasible only if the patient size is below a particular threshold. However, in routine CT scans, it is difficult to determine the optimal $\mathrm{kV}$ setting for individual patient, simply because of the complex relationship among $\mathrm{kV}, \mathrm{mAs}$, dose, contrast, and image noise. To solve this problem, Siemens recently introduced a novel tool, CARE kV, which allows the optimal $\mathrm{kV}$ to be determined automatically for each individual patient and each specific exam. Basically, CARE $\mathrm{kV}$ uses information obtained from the topogram to determine the optimal $\mathrm{kV}$ which is used to maintain image quality (e.g., contrast-to-noise ratio). Together with AEC described above, this tool can be beneficial in optimizing and reducing radiation dose to each individual patient.

2.1.6. Image Reconstruction. Filtered back projection (FBP) is still the most common reconstruction method used in CT. Over the past two decades, iterative reconstruction (IR) method has been shown to be superior to conventional FBP method in terms of image noise and spatial resolution [5356]. The improvement in image quality can directly lead to 
Siemens: SAFIRE

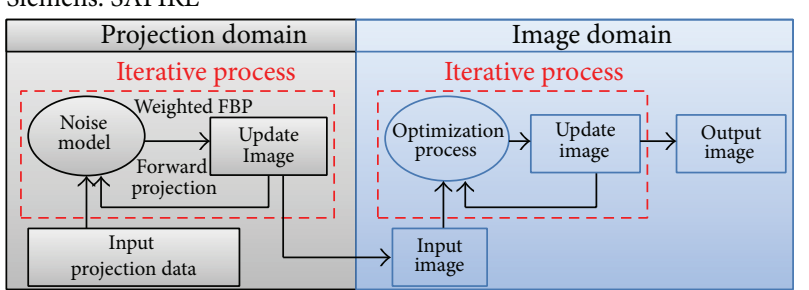

Toshiba: ADIR 3D

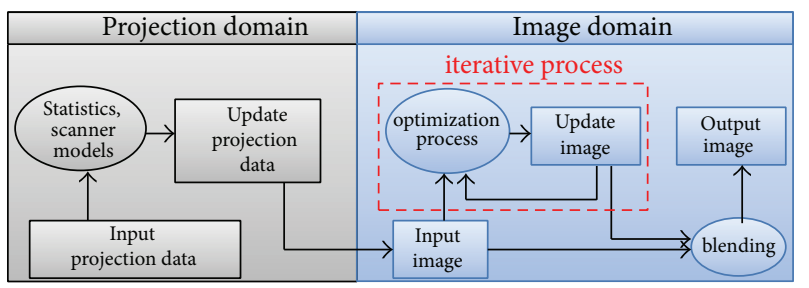

Philips: iDoese ${ }^{4}$

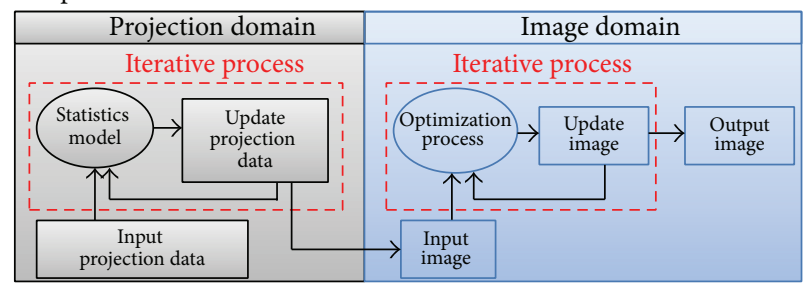

GE: ASIR

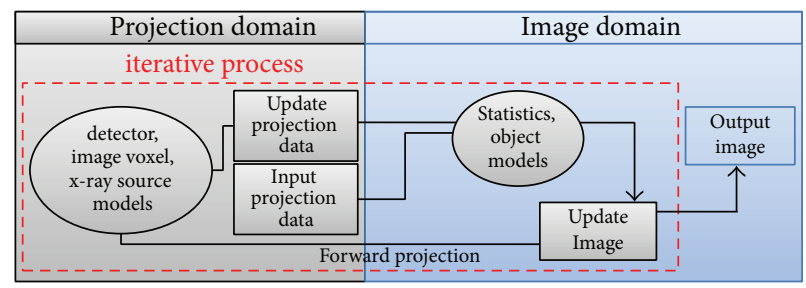

FIGURE 2: The schematic of four CT manufactures' IR algorithms: Siemens' Sinogram Affirmed Iterative Reconstruction (SAFIRE), Philips' iDose ${ }^{4}$, Toshiba's Adaptive Iterative Dose Reduction 3D (ADIR 3D), and GE's Adaptive Statistical Iterative Reconstruction (ASIR). Both SAFIRE and iDose $^{4}$ have a similar iterative correction process operating in the image domain. In the projection domain, SAFIRE performs a projection data correction via forward and backward (i.e., weighted FBP) projection; however, iDose $\mathrm{e}^{4}$ directly corrects projection data. ADIR $3 \mathrm{D}$ performs a noniterative correction in the projection domain and an iterative correction in the image domain. Also, a weighted blending is applied to the input image (i.e., after projection data correction) and the output image (i.e., after iterative process). Unlike the other three IR algorithms, ASIR performs an iterative correction process by comparing synthesized projection data to measured projection data.

a significant reduction in patient dose. However, IR method is rarely used in routine CT exams, mainly owing to the high computational time required for modeling the system geometry and incorporating physical effects (e.g., noise, scatter, beam hardening, and detector response). Due to advances in hardware [57-59] and renewed emphasis on CT dose reduction, fast IR methods have recently been developed and implemented for radiation dose reduction. Now, IR methods are available on many CT scanners from four major CT manufactures with different algorithms (Figure 2), to perform corrections in both the projection and the image domain. They are applied to achieve noise reduction and artifact reduction as well as to preserve structural edges.

Another important image reconstruction technique toward the goal of reducing radiation dose is the use of incomplete projection data, either to acquire a small number of projection angles or to use the truncated projection data. The first strategy is the sparse-view CT and the second one is the interior CT [60]. With the recent introduction of compressed sensing (CS) in CT [61, 62], it is possible to perform image reconstruction from a small number of projections while still maintaining image quality [63-67]. The basic concept of CS is to recover a signal or an image from measured data with sampling rates below the Nyquist criterion. To accurately restore the signal from the undersampled data, computational load may be still too high for practical use. Thanks to the hardware-accelerated image reconstruction technique [57-59], it is expected that fast CS-based reconstruction methods will be introduced to allow an ultra-low dose CT scan in clinical routine. On the other hand, interior $\mathrm{CT}$ uses the projection data passing through a region of interest (ROI) to reconstruct image. In practical, this technique can lead to a dose reduction in patients with cardiac or breast CT scans. The exact ROI reconstruction methods from such truncated projection data have been proposed $[68,69]$. Unlike approximate reconstruction methods [70, 71], the new analytical reconstruction method called differentiated backprojection (DBP) was shown to have a potential to exactly solve the ROI reconstruction [60]. Despite the fact that these reconstruction methods have a great potential to reduce CT radiation dose, there are some assumptions needed to be satisfied. For example, some prior knowledge about the object is required.

2.2. Dual-Energy CT (DECT). Due to the recent advances in CT technology including sandwiched detectors $[72,73]$, rapid $\mathrm{kV}$ switching [74], and dual-source and detector [7577], there is a renewed interest in DECT. To date, DECT has many clinical applications such as bone removal with plaque highlighting, quantification of iodine concentration, visualization of tendons and ligaments, and characterization of renal calculi [78-80]. Using the material-specific information provided by DECT data, virtual noncontrast (VNC) images can be generated by removing iodine content from the dualenergy contrast-enhanced CT images. As a result, DECT has the advantage of omitting one unenhanced (i.e., precontrast) CT scan, thus reducing the overall radiation dose. For example, VNC images generated from either nephrographicor excretory-phase DECT data may have the potential to replace unenhanced $\mathrm{CT}$ images for detection of urinary stone $[81,82]$, evaluation of haematuria [83] and characterization of renal masses $[84,85]$. One recent study showed that iodine map generated from DECT can be used to differentiate between benign and malignant mediastinal tumors [86]. Moreover, iodine concentration provided by DECT strongly correlated with the maximum standardized uptake value of 
${ }^{18}$ FDG PET that was commonly used functional imaging technology [87]. More importantly, initial results showed that DECT can be used as an imaging biomarker for predicting the therapeutic benefit in patients with gastrointestinal stromal tumors [88]. We believe that DECT is capable not only of providing functional imaging information but also of giving dose-saving opportunities.

2.3. Perfusion $C T(P C T)$. PCT is an existing imaging technique that has been used to determine tissue perfusion from dynamic contrast-enhanced CT data. Due to the feasibility of rapid acquisition of data (i.e., multislice spiral CT) and the availability of commercial software, clinical applications of PCT in acute stroke, cardiology, and oncology have continued to increase $[89,90]$. Recent results showed that PCT positively correlated with tumors in the study of animal models $[91,92]$. With PCT, it is also possible to detect and predict tumor response to chemotherapy and radiation therapy [93]. To obtain perfusion parameters (e.g., blood flow and volume and vascular permeability), sequential acquisition of CT data after a bolus injection of contrast agent is required. Typical image acquisition time is about 30 seconds or longer, depending on organ or tissue being scanned, so the radiation dose of a PCT scan is much higher than that of a routine CT scan [94]. As a result, methods that allow for a significant reduction of CT dose without degrading image quality are highly desirable. Since PCT contains temporal information, reconstruction algorithms that incorporate temporal filtering techniques have been proposed to reduce image noise [95-99]. Indeed, such reconstruction algorithms can allow a significant reduction in image noise, thereby having a large potential to reduce the radiation dose in PCT. One alternative strategy to reduce radiation dose in PCT is to optimize scanning parameters such as total scanning time and sampling interval $[100,101]$. With the implementation of dose-reduction technologies, PCT will be increasingly used in clinical practice.

2.4. Summary. We would like to summarize the CT dose reduction technologies so that it would be easier to understand their feasibilities.

(i) To accomplish the principle of ALARA, each CT scan should be customized for the patient's age, weight, size,and imaging task. Individual customized CT scanning techniques used in current clinical CT scanners include examination-specific bow-tie filters, adaptive collimator, and automatic tube potential selection with tube current modulation.

(ii) CT detectors with integrated electronics or a garnetbased scintillator have a great potential to reduce noise, thereby improving image quality and dose efficiency. CT scanners equipped with high-rate photoncounting detectors will be available in the near future for further dose reduction.

(iii) As provided by four major CT manufactures, iterative reconstructions result in significant dose reduction and improved image quality over conventional filtered back projection reconstruction methods. New reconstruction methods for sparse-view CT and interior $\mathrm{CT}$ will be an alternative to dose reduction.

\section{Imaging Acceleration in Magnetic Resonance Imaging}

Since the introduction of MRI in the early 1970's [102], its techniques have been much improved from hardware to image reconstruction. Due to the intrinsic physical limitation of MRI, the speed of data acquisition is always the problem comparing to CT. Hence, a number of acceleration techniques have been developed over the past four decades and the shortened acquisition time has greatly expanded clinical applications of MRI, especially for dynamic or timeresolved MRI, such as perfusion imaging [103-106], contrastenhanced MR angiography [107-111], functional MRI [112, 113], and cardiac function examinations [114-117]. Those acceleration techniques could be divided into two categories: parallel imaging and dynamic acceleration. Besides, CS, which is beyond the limitation of Nyquist criterion [61, 62], inspires the MR physicists and the radiologists for even faster scans.

3.1. Parallel Imaging. The typical acquisition time of an $\mathrm{MR}$ image is the product of the repetition time (TR), number of phase-encoding lines $\left(N_{\mathrm{PE}}\right)$, and the slice number $\left(N_{S}\right)$ (Figure 3). In order to shorten the scan time, the reduced phase-encoding $k$-space lines are generally acquired, which would either sacrifice the image resolution or result in the aliasing artifacts. Parallel imaging technique, which combines the design of the phase-arrayed coil and various image reconstruction algorithms, makes the faster data acquisition possible. Hence, the common features of the parallel imaging include the under-sampled $k$-space data and the phasearrayed coils, which provided additional spatial information for image reconstruction to solve the aliasing patterns. The reconstruction algorithms could be divided into SENSE and GRAPPA family, where the undersampled data are reconstructed in image domain and $k$-space, respectively. Besides, the multislice acceleration acquisition is a kind of extension of parallel imaging and we would discuss it after the two conventional techniques.

3.1.1. Generalized Autocalibrating Partially Parallel Acquisition (GRAPPA). GRAPPA [118] technique originated from SMASH [119]. SMASH technique reconstructed the MR images from filling in the missing phase-encoding lines by using the phased-array coil, whose elements are parallel to the phase-encoding direction. Because the acquired signals from different surface coils imply the spatial sensitivity information in the meantime, they could be used to generate multiple datasets, which are called harmonics, by using linear combination with different weightings to complete the whole $k$-space. The restored full $k$-space was then Fouriertransformed to obtain the reconstructed image (Figures 2 and 3 in [119]). Depending on the concept of linear algebra, $N$ components could form maximal $N$ linearly independent combinations and result in maximal acceleration rate of $N$. 


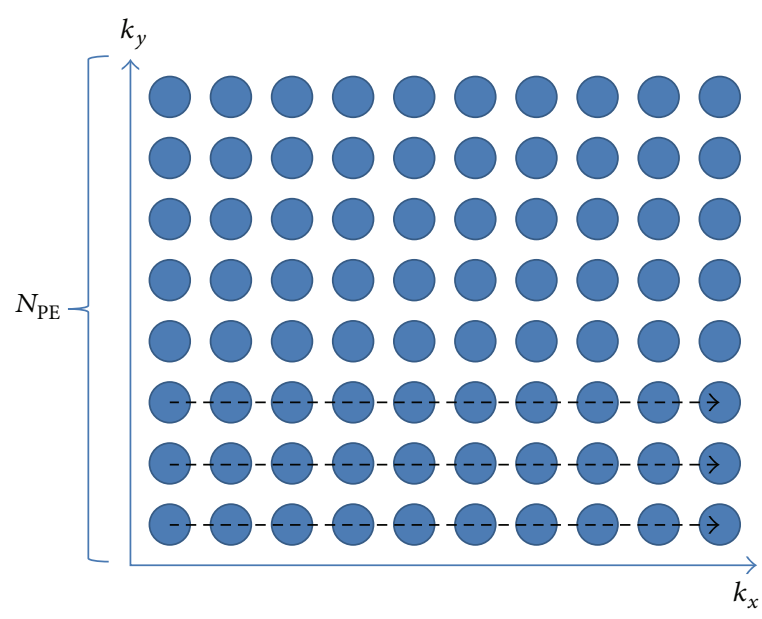

$k_{x}$ : frequency encoding direction

$k_{y}$ : phase encoding

$k_{z}$ : slice encoding (for 3D imaging) or slice direction (2D)

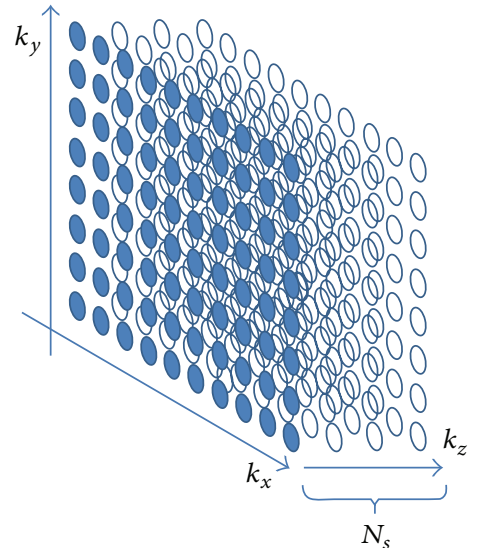

FIGURE 3: Illustration of three-dimensional $k$-space in MRI. Generally, the sampling time of $k_{x}$ was less than repetition time (TR), so the acquisition time of a set of image could be written as TR $\times N_{\mathrm{PE}} \times N_{S}$.

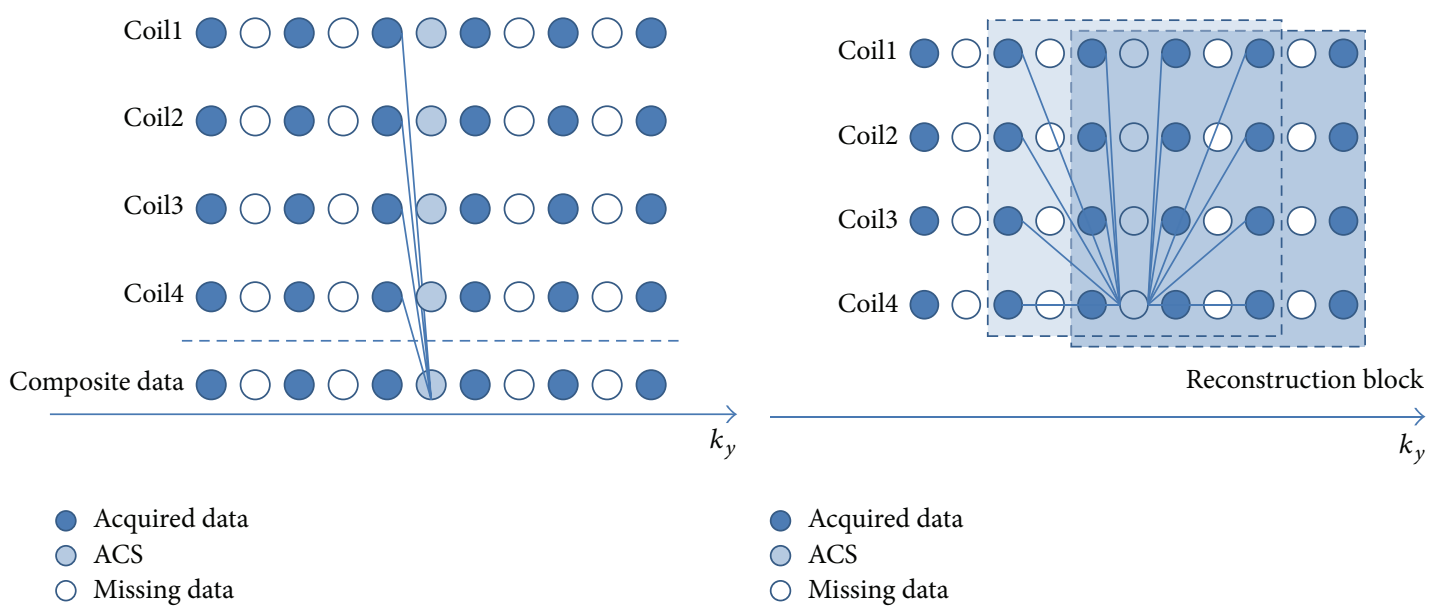

(a) (b)

FIGURE 4: (a) AUTO-SMASH or VD-AUTO-SMASH reconstruction process for the acceleration rate of 2. Each $k$-line in a single coil was composited to fit the ACS line, and the relationship was applied to fill in the missing $k$-lines. The procedures constructed the whole $k$-space and then the reconstructed images were obtained. SMASH did not additionally acquire ACS. It used the estimated coil sensitivities to composite the missing data. (b) GRAPPA used multiple lines from all coils to fit a line in one coil. The reconstructed images from each coil were obtained after the $k$-space acquired from each coil was fully restored. The uncombined images were merged using sum of squares or other adaptive algorithms. The concept of reconstruction block made GRAPPA flexible using different "blocks" to fit the ACS lines for better performance. For example, at least there were three blocks used for ACS lines fitting, which would provide a more accurate outcome.

However, the harmonics are generally less than the total number of array components due to the geometrical constraints.

However, the major pitfall of SMASH is its high dependence on accurate coil sensitivity function, which might be easily affected by coil positions, subjects, and magnetic field inhomogeneities. Hence, some other methods accompanying the acquisition of autocalibration signals (ACS) near the central $k$-line were developed for the sensitivity function calibration, such as AUTO-SMASH [120] and VD-AUTOSMASH [121]. Furthermore, GRAPPA provided a more general form to reconstruct the full $k$-space data using multiple blocks in sliding manners rather than SMASH and its analogies from adjacent acquired $k$-lines (Figure 4 ). It is more flexible to fit the ACS from the acquired signals and restores the missing $k$-space data more precisely. The number of ACS is another factor to influence the reconstruction quality. Generally speaking, the more ACS acquired, the more accurate GRAPPA weightings obtained, which result in less artifacts but reduced the acceleration efficiency. GRAPPA is a more robust method and less relies on the coil sensitivity 


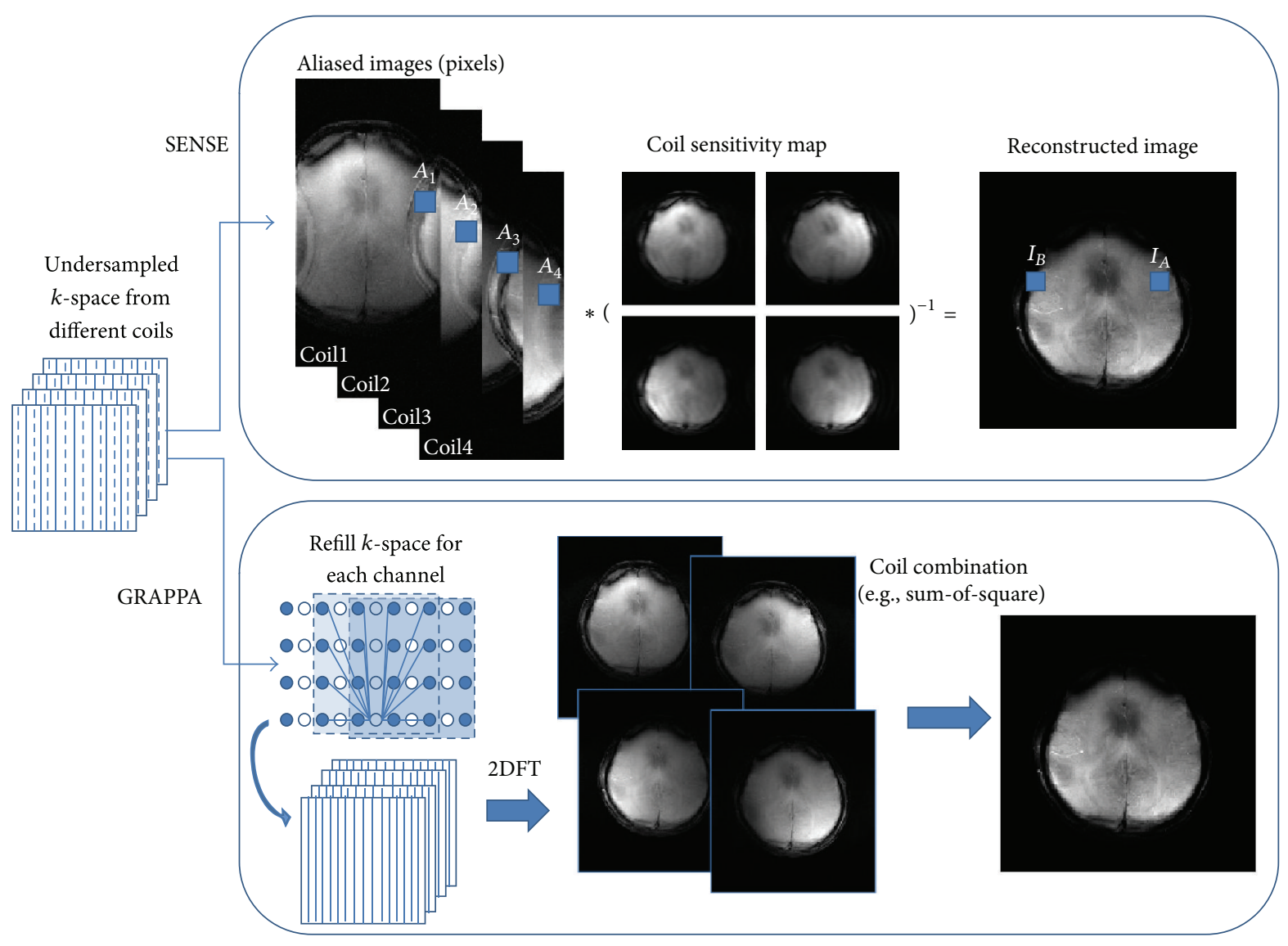

FIGURE 5: The reconstruction process of SENSE and GRAPPA. The example used a four-channel array coil for the acceleration rate of two. Each of the aliased pixels $\left(A_{1}, A_{2}\right.$, and so on) came from $I_{A}$ and $I_{B}$ with different weightings depending on the coil sensitivity values. It could be represented as $A_{i}=I_{A} C_{A i}+I_{B} C_{B i}$, where $A$ was the aliased pixel, $I$ was the intensity of the unaliased pixel, and $C$ was the coil sensitivity weighting from the corresponding location. It could be possible to reconstruct the data by finding the inverse matrices of coil sensitivity maps, which could be written as $A C^{-1}=I$. GRAPPA reconstructed the undersampled $k$-space using ACS fittings to calculate the GRAPPA weightings and refill the missing lines from each coil. Each restored full $k$-space was transformed to obtain the single-coil images, which were then combined together to finalize the reconstruction.

maps, so that the cardiac, lung, and abdominal applications become feasible. Besides, it could be also applied to $3 \mathrm{D}$ imaging $[122,123]$.

3.1.2. Sensitivity Encoding (SENSE). SENSE [124] technique began from the aliased images resulting from the undersampled $k$-space acquisition. Considering the case of acceleration factor $R$, each aliased pixel from different phase-arrayed coils should be divided into $R$ partitions with different weightings relating to coil sensitivities (Figure 5). Hence, the coil sensitivity map, which is usually obtained from a lowresolution prescan at the beginning of the image acquisition, is the prior information for SENSE reconstruction. Since SENSE reconstruction much relies on the coil sensitivity map, the inaccurate sensitivity map would lead to residual aliasing artifacts. One of the most important factors to sensitivity map is SNR. Low SNR regions, for example, lung images, have a difficulty in determining the coil sensitivity distribution.

The common features for the two parallel imaging families are the reduction of SNR and the residual aliasing. The significant difference is the reconstruction domain: $k$-space for GRAPPA and image domain for SENSE (Figure 5). The SNR reduction of parallel imaging could be generalized to

$$
\mathrm{SNR}_{\mathrm{PI}}=\frac{\mathrm{SNR}_{\mathrm{fs}}}{g \sqrt{R}},
$$

where fs means full sampling, $R$ is the acceleration factor, and $g$ is the factor relating to some coil properties, such as geometry design $[125,126]$. The meanings of $g$-factor in GRAPPA and SENSE are different, but it is mostly larger than one. Hence, many dedicated surface coils were developed, such as breast coils, spine arrays, and head coils, to reduce the noise enhancement. In addition, the ill-conditioned problem also leads to the loss of SNR. Thus, the use of regularization in SENSE [127-129] as well as GRAPPA [130] was developed. Though there are some compromises in parallel imaging, it is still routinely used in various clinical applications [131] due to improvements in acquisition time, spatial or temporal resolution, and image quality such as reduction of motion artifacts and EPI geometric distortion. 


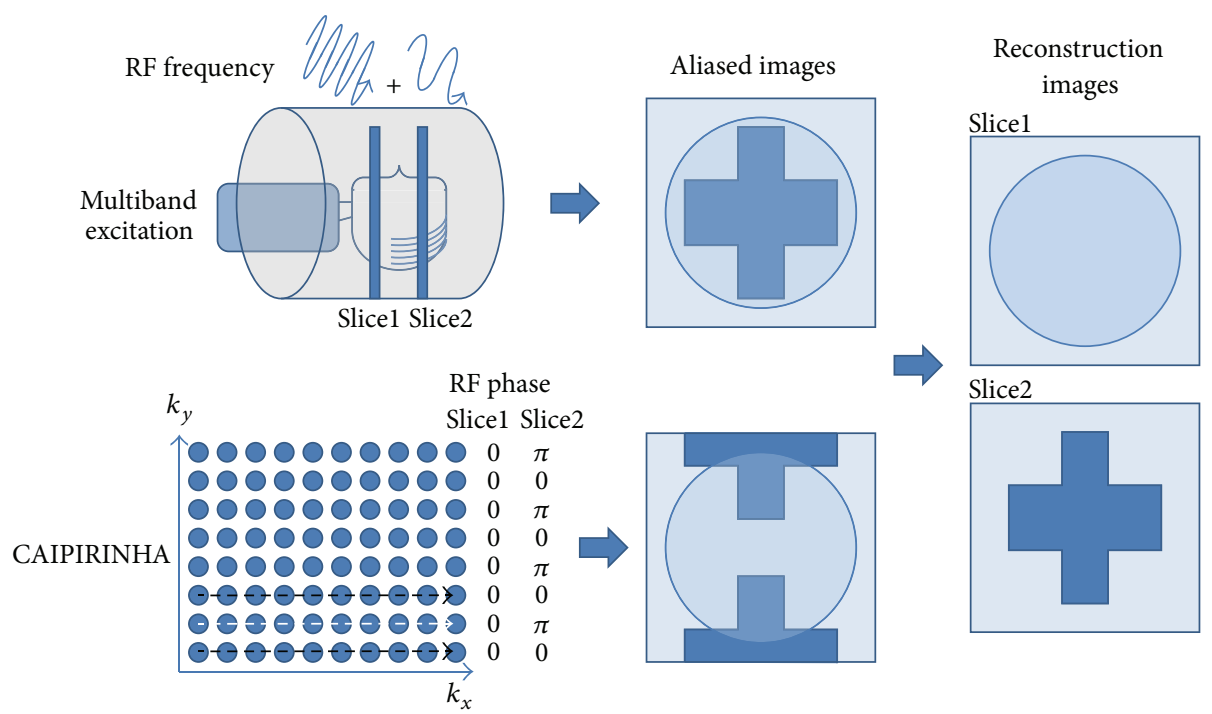

FIGURE 6: Schematic description of the differences between MB excitation and CAIPIRINHA. The slice selection gradient would be applied during the RF excitation, where the RF frequency and the slice resonant frequency were the same. Since MB excitation and CAIPIRINHA excited multiple slices simultaneously, the bandwidth of RF excitation would become wider than the single slice excitation. MB excitation modulated the RF excitation pulse with several frequencies to excite multiple slices, so the acquired images would overlap together without FOV shifting (the upper row). Considering the bandwidth of frequency encoding and the removal of the artifacts, the excitation slices should have gaps with several centimeters depending on the gradient strength. Besides, the pixel skew was another factors controlled by the gradient strength [20]. CAIPIRINHA, which also excited multiple slices simultaneously, controlled the aliasing pattern by phase-modulated RF pulses. In the figure, for example, the phase of RF pulse in the second slice was interlacedly altered to shift the overlapping patterns with FOV/2 (the lower row). The black dashed arrow indicated the phase-encoding line without phase alteration in both slices, and the white one did the phase alteration of $\pi$ only in the second slice. It could improve the $g$-factor to increase the SNR in image reconstruction. The technology could get rid of the pixel skew in MB excitation and make adjacent slice excitation feasible.

3.1.3. Multislice Excitation. GRAPPA and SENSE could be extended to $3 \mathrm{D}$ imaging $[122,123]$, which is slab excited without additional RF modification. An alternative idea for acceleration is the multislice simultaneous excitation with the sharing data acquisition schemes. The RF excitation type could be divided into frequency modulation, such as multiple band (MB) excitation $[20,132]$, and phase cycling, such as CAIPIRINHA [133] (Figure 6).

Both techniques have some intrinsic limitations. In MB excitation, the frequency offsets between slices should be at least equal to or larger than the slice bandwidth to ensure the successful reconstruction from the slice aliased images. Thus, two solutions could be applied: either to increase the gap between the excited slices at around several centimeters or to strengthen the slice selection gradient, which would result in the pixel skew. CAIPIRIHNA, on the other hand, controlled the aliasing pattern using phase modulated multislice RF excitation pulses to avoid the pitfalls in MB excitation technique and improved the image quality of 2D-GRAPPA or 2DSENSE reconstruction resulting from $g$-factor related noise enhancement (Figures 6 and 7 in [133]). Furthermore, instead of providing phase modulated RF pulses, the shifted aliasing patterns could perform more easily in $3 \mathrm{D}$ volume images via changing the encoding strategy (2D CAIPIRINHA) [21]. Hence, various developments in sequences $[134,135]$ and clinical applications $[136,137]$ were demonstrated more easily. However, neither CAIPIRINHA nor conventional MB excitation techniques are not applicable to single-shot echo planar imaging (EPI), since the former method uses shot-by-shot RF modulation and the latter one suffers from high $g$-factors due to the hardware limitations. Hence, taking the concept of inclined readout [20,132], the blipped wideband [138] and blipped-CAIPIRINHA [139] were proposed to make simultaneous multislice EPI and diffusion imaging feasible with reasonable $g$-factors. The significant advantages are that the temporal resolution could be increased for fMRI and the acquisition time could be much reduced for DSI or Q-ball imaging.

$\mathrm{MB}$ and CAIPIRINHA, generally in charge of the slice direction acceleration, could incorporate with the conventional parallel imaging techniques to fully achieve 2D acceleration in a better performance. Since all the techniques are based on the reduced $k$-space sampling for acceleration, the residual artifacts should be carefully dealt with.

3.2. Dynamic Acceleration. The dynamic MRI introduces temporal information into the acquisition, so it provides an additional dimension for acceleration. Only the signals of certain regions were varied with time in dynamic imaging; for example, contrast-enhanced angiography shows the signal intensity change in vessels and the rest parts are almost static. Hence, there are lots of redundant acquired data during the examination, and it could be well manipulated to reduce the scan time for each time frame and to increase the temporal resolution. The first idea came to view sharing in $k$-space. 

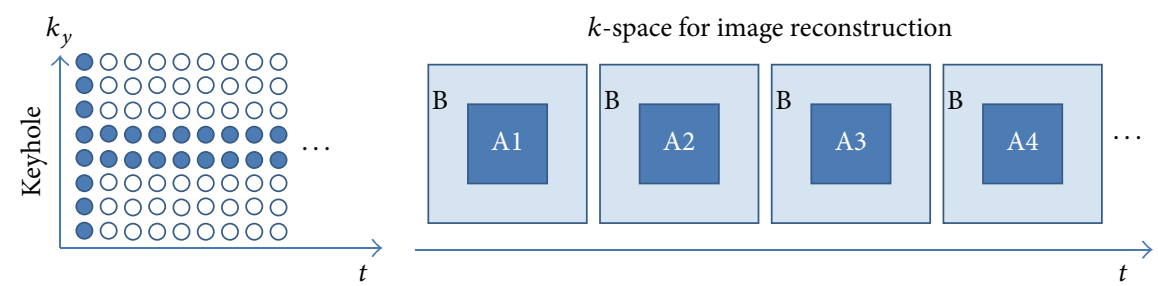

(a)
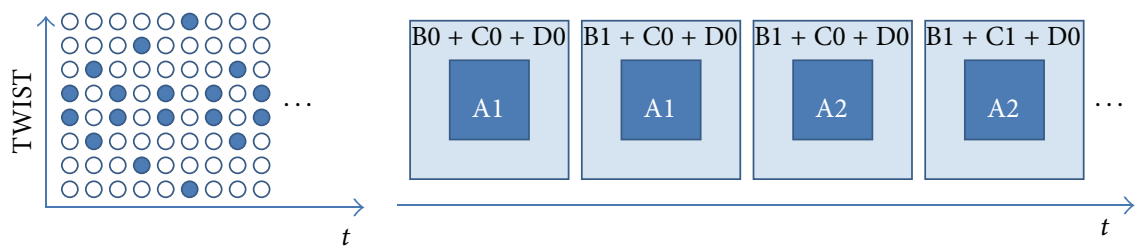

(b)
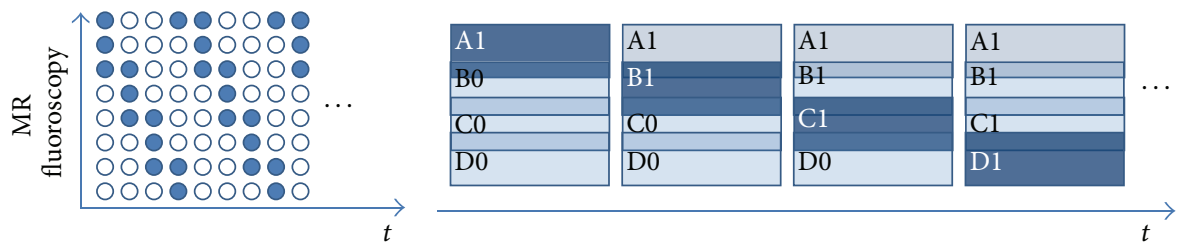

(c)

Figure 7: The sampling features in view-sharing technologies: Keyhole, TWIST, and MR fluoroscopy. (a) Keyhole acquired full $k$-space at the beginning or the end of the dynamic scan. Only central $k$-space (A1, A2, and so on, where the number indicated the times of update) was renewed at each time frame and the rest was kept the same for image reconstruction. (b) TWIST updated the central $k$-space information more frequently. The outer $k$-space was divided into several subregions, for example, three (B, $\mathrm{C}$, and $\mathrm{D})$ in this case, and only one of them was updated at each time frame interleaved by the renewal of the central $k$-space. (c) MR fluoroscopy updated the $k$-space sequentially and uniformly everywhere. Part of the information was acquired at each time frame and updated to $k$-space for dynamic image reconstruction.

3.2.1. View Sharing. Taking the advantages of the similarity of the adjacent time frames, several view sharing technologies were developed since the late 1980s, including MR fluoroscopy [140], Keyhole [141], BRISK [142], and TWIST [143, 144]. The major differences among the technologies are the manners on dynamical $k$-space data replacement and the data recovery.

MR fluoroscopy periodically updates the $k$-space data so that the refreshed rate of each part is the same. Because partial $k$-space is acquired at each time point, the data recovery should include the information from some previous adjacent time frames and the latest acquired one to obtain full $k$-space data and then convert to images. The technique is also called moving average or sliding window reconstruction. However, it is not efficient to update the $k$-space uniformly due to most of the useful information locating on central $k$-space. Hence, Keyhole pushes the idea into a limit, where the peripheral $k$ space data are only acquired once at the beginning or the end of the dynamic scan and the center is refreshed at every time frame. BRISK and TWIST are the compromise between the two extreme cases. They divide $k$-space into different zones, where the central one and the one of the peripherals are alternatively updated (Figure 7).

These techniques refill the missing $k$-space using the temporally closest data. The process is equal to the dynamic images interpolation, which would smooth the dynamic response curves similar to applying low-pass filters. Hence, some high temporal frequency information would get lost, so that the acceleration rate and the view-sharing methods should be carefully chosen depending on different applications. However, they did not really take the advantages from the additional dimension, time, for acceleration, so more sophisticated approaches were developed.

3.2.2. Unaliasing by Fourier-Encoding the Overlaps Using the Temporal Dimension (UNFOLD). UNFOLD [113] was the very early technique taking the advantages of spatial and temporal information for acceleration. It converted the acquired $k$-space data with temporal information ( $k$ - $t$ space) into $x$ - $f$ space to observe the dynamic patterns, so that we could more effectively exploit the $x$ - $f$ space for higher acceleration rates (Figure $8(\mathrm{a})$ ). The " $x$ " in $x$ - $f$ space represents the image domain, not exactly the $x$-axis, and generally the undersampled $k$-space is along the phase-encoding direction, which is usually the $y$-axis. The concept of UNFOLD is similar to 2D CAIPIRINHA [21], where the images become less aliasing by modifying the sampling strategy (Figure 8(b) and Figure 3 in [21]). The major difference is the second acceleration dimension of 2D CAIPIRIHNA along slice encoding direction and that of UNFOLD temporally. However, a prerequisite of UNFOLD technique is that 


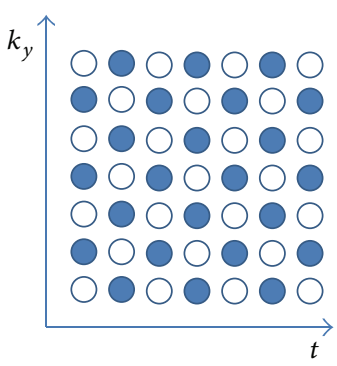

UNFOLD

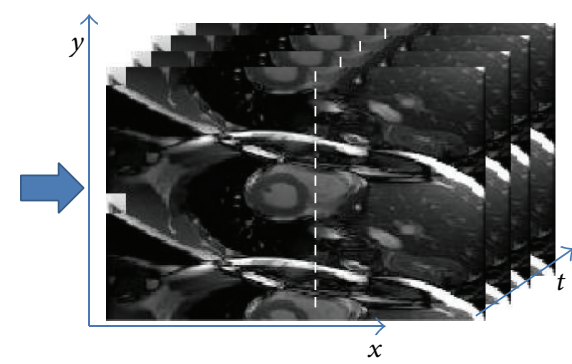

(a)
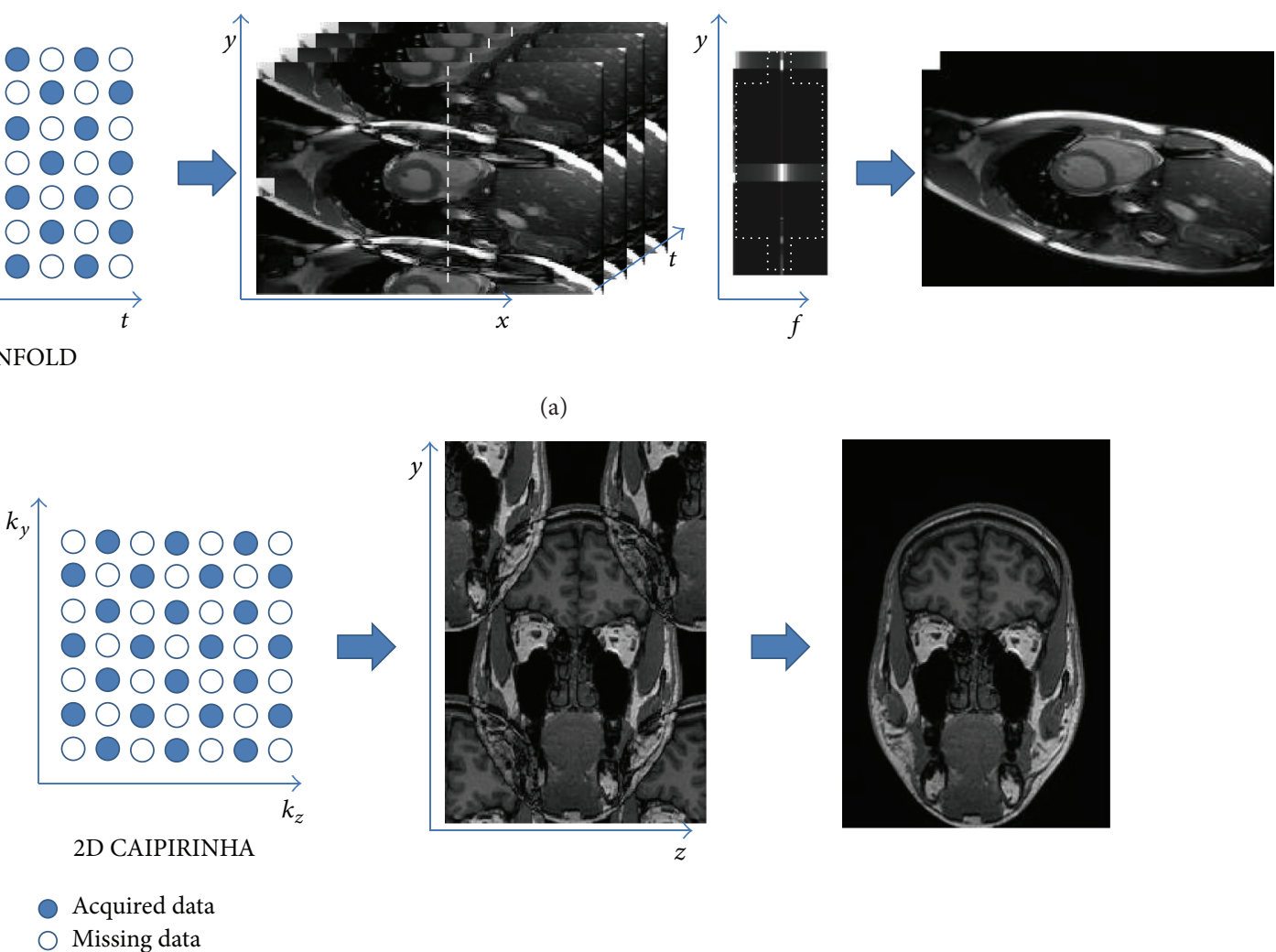

(b)

FIGURE 8: The similarity and difference between UNFOLD and 2D CAIPIRINHA. Both techniques used two-dimensional accelerations, where the second domain in UNFOLD is time and that in 2D CAIPIRINHA is the slice encoding direction $\left(k_{z}\right)$. (a) UNFOLD transformed the dynamic aliased images to the $x$ - $f$ space, where the information was more concentrate. The $x-f$ aliasing patterns could be shifted by changing the sampling patterns. Hence, the space was suitable to reduce the aliasing patterns and reconstruct the images by applying appropriate filters (the dashed cross region). (b) 2D CAIPIRINHA also changed the sampling patterns to shift the aliased images, which could reduce the $g$ factor and improve the SNR in reconstruction. The performance would be better than that in conventional $2 \mathrm{D}$ parallel imaging techniques [21].

the aliased $x$ - $f$ space should not overlap in major frequency components. Then the aliasing artifacts could be removed in $x-f$ space by applying appropriate filters to fully reconstruct the images.

The limitation of acceleration rate in UNFOLD depends on different applications. The larger the portion of dynamic region within an FOV, the less the acceleration rate achieve. Hence, the acquisition time of fMRI could be more efficiently reduced than that of cardiac imaging.

\subsection{3. $k$-t Family. Depending on the concept from UNFOLD} technique, $k$ - $t$ BLAST and $k$ - $t$ SENSE [145] were developed to enhance the robustness of the achievable acceleration by acquiring the central part of $k$-space at the beginning as the training data before the undersampled dynamic $k$-space acquisition. Hence, the whole acquisition is divided into two parts: the training scan, providing the low spatial resolution images without aliasing artifacts, and the undersampled scan, containing the full spatial and temporal information but with fold-over artifacts. $k$ - $t$ BLAST and $k$ - $t$ SENSE use the training data as prior information to understand how to resolve the aliasing patterns and to obtain the fully-reconstructed dynamic images. $k$ - $t$ SENSE, combining the parallel imaging technology, was an extension of $k$ - $t$ BLAST, and both technologies reconstruct the images in $x$ - $f$ space (Figure 9). Because $k$ - $t$ methods introduce temporal information to increase the degree of freedom, their performance, especially in noise reduction of the reconstructed images could be much better than the traditional parallel imaging at high acceleration factors.

$k$ - $t$ GRAPPA $[146,147]$ reconstruct the data directly in $k$ $t$ space without transforming to $x$ - $f$ space. ACS is acquired to determine the weightings for $k$-space reconstruction as GRAPPA does. $k$ - $t^{2}$ GRAPPA [148] is an extension of the series and scatters the central $k$-space acquisition into different time frames. Hence, the reconstruction should firstly use $k$ - $t$ GRAPPA to refill the undersampled central $k$-space and then to restore the full $k$-space data. $k$ - $t$ PCA [103] is another kind of extension, which reconstructed the images using principal components (PC), instead of temporal frequency. Thus, it uses $x$-PC space for data reconstruction. Taking the advantages of mathematical properties of principal component analysis (PCA), $k$ - $t$ PCA could even reduce the artifacts for higher acceleration rates. 

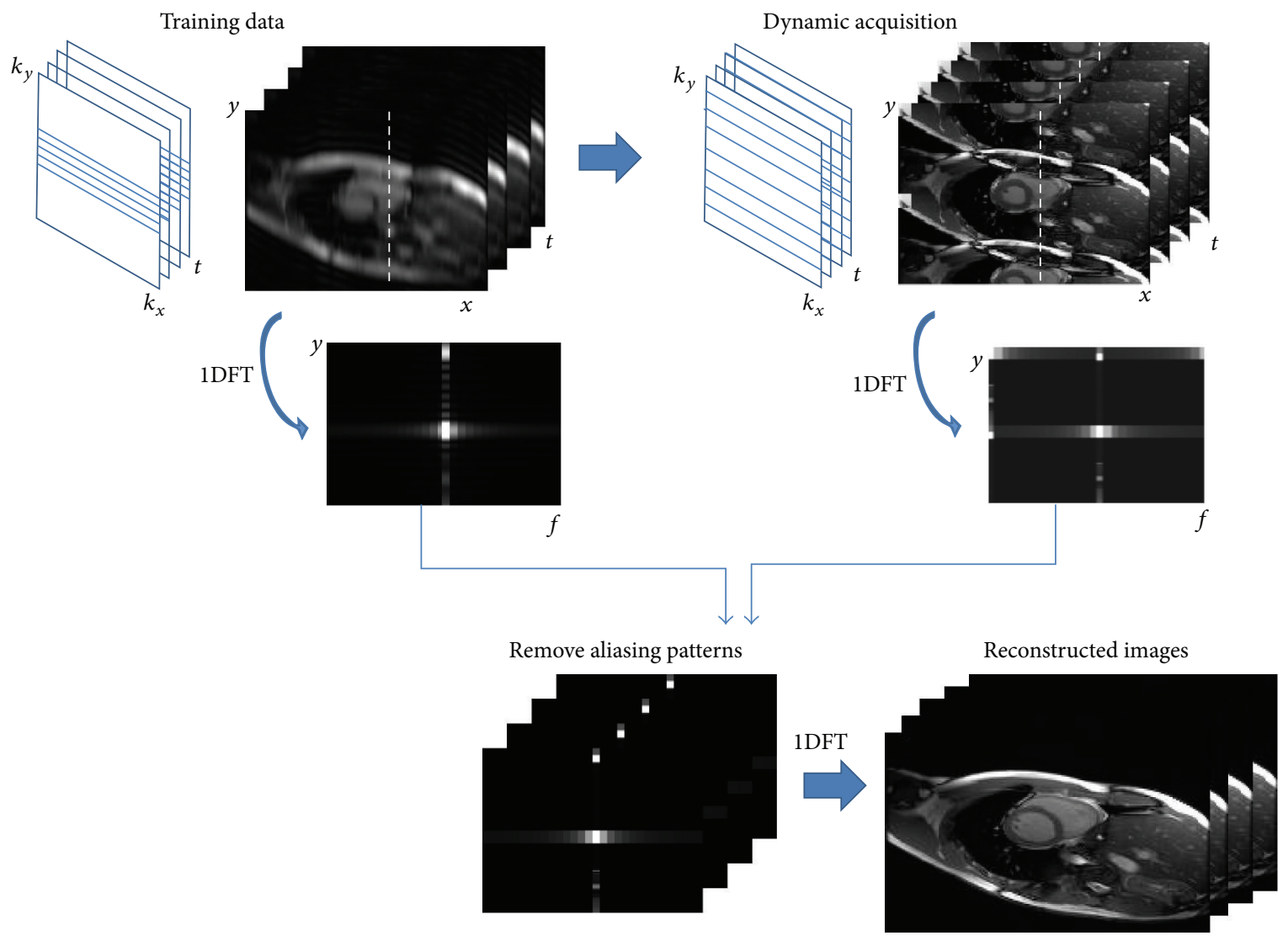

FIGURE 9: $k$ - $t$ BLAST for dynamic imaging acceleration. The data acquisition is divided into two parts, training data (low resolution images) and dynamic acquisition (undersampled $k$-space images). The $x$ - $f$ space of the training data is used to preliminarily understand the features of the dynamic scan, and then the undersampled data could be involved for image reconstruction without applying any determinate filters. An alternative method was to embed the central $k$-line acquisition in every time frame and the data were split into two parts as the description above when doing image reconstruction.

3.3. Compressed Sensing (CS). CS [62] was first proposed in the field of information theory and is applied to MRI with the sampling rate beyond the Nyquist criterion. The sampling number could be much reduced in CS, as a result, the acquisition time becomes much shorter. There are three basic requirements for the CS approach, including the sparsity in a known transform domain, the incoherence of the undersampled artifacts and the nonlinear reconstruction.

Most of the MR images are not sparse from image point of view except angiography. However, sparsity is the most fundamental requirement to represent whether the data are compressible or not. Many different kinds of images are sparse in discrete cosine transform (DCT) and wavelet transform, which are the cores of image or video compression technologies. Medical images share the same property of sparsity, so that the DCT and the wavelet transform are both good for brain and angiogram which were capable to use only $5 \%$ to $10 \%$ largest transform coefficients for reconstruction [149]. Conventional undersampled $k$-space was coherent, that is, the sampled $k$-space was skipped periodically and the reconstructed images resulted in regular aliasing patterns. On the other hand, the incoherent undersampled $k$-space would lead to the noise-like artifacts, so the dealiasing problems could be converted to a denoise problem. The two aliasing patterns show significant differences in Figure 1 of [150]. Since the sampling strategy has destroyed the linear transformation relations between two domains ( $k$-space and image), some nonlinear reconstruction methods should be applied to iteratively approach the solutions, such as conjugate gradient method [151].

The undersamples along frequency encoding direction could not actually reduce the scan time in MRI and it was not feasible to achieve the fully randomized sampled $k$-space. Several encoding schemes, such as radial and spiral [110, 152, 153], could approach the variable-density sampling, which results in more incoherent aliasing patterns. For morphological or static images, 2D imaging acquisition is not preferred because the randomly undersampled dimension is limited. It is more attractive on three-dimensional imaging or dynamic images, because there are two nonfrequency encoding planes $\left(k_{y}-k_{z}\right.$ or $\left.k_{y}-t\right)$, which would achieve random undersampling more easily. However, one of the features of $k$-space should be taken into consideration when doing randomized undersampling: the energy of $k$-space is concentrated in 
the central region. Hence, the central $k$-space should be less undersampled than peripheral regions to keep the image quality and to accelerate the data acquisition more efficiently.

CS has been demonstrated in various applications with the combination of parallel imaging techniques in static images $[149,154,155]$. Furthermore, there were also technologies developed with the combination of the concepts from $k$ - $t$ BLAST and incoherent sampling, such as $k$ - $t$ SPARSESENSE $[156,157]$ and $k$ - $t$ FOCUSS $[158]$, and dynamic MRI using CS was applied to cardiac imaging $[159,160]$ and fMRI $[161,162]$. However, the computational time is still too long for daily clinical use. Thus, the improvement in algorithms and computer hardware, which shorten the postprocessing time, would bring CS more feasible.

3.4. Summary. We would like to summarize the MR acceleration technologies so that it would be easier to distinguish the differences and understand their feasibilities.

(i) Parallel imaging uses the multiple-channel surfacearrayed coils to reduce the $k$-space sampling. The reconstruction methods are divided into GRAPPA and SENSE two families, which are commonly used in clinical routines.

(ii) Multislice excitation, the extension of parallel imaging, could be divided into frequency modulation (MB) and phase modulation (CAIPIRINHA) for the acceleration along the slice direction with better performance.

(iii) Temporal domain is involved in dynamic acceleration methods, such as UNFOLD and $k$ - $t$ BLAST. UNFOLD applies an appropriate filter to remove the aliasing patterns, where $k$ - $t$ BLAST does it via the low-resolution training data at the beginning of the dynamic acquisition.

(iv) Compressed sensing MRI brings the acquisition beyond the Nyquist criterion limitation both in static and dynamic images.

(v) All the technologies above are compatible to parallel imaging techniques.

With the appropriate combination of these techniques, we could foresee the huge leap in MR acceleration in the near future.

\section{Conclusion}

CT dose consideration and MR acquisition could be viewed as the two obstacles during their development. We have seen many techniques developed to solve the problems, to broaden their applications, and to push the diagnostic clinical imaging into molecular imaging level. Besides, their intrinsic superiorities are kept to measure the physiological parameters, such as blood flow, blood volume, and $T_{1} / T_{2}$ parameters or diffusion imaging specified by MRI. The information has been investigated for oncology imaging, cancer treatment followup, neurodegenerative diseases, and so on. Due to the growing knowledge of the human multiple "-omes," such as genome, proteome, and metabolome, more information is included to analyze the correlation between the -omic data and the clinical imaging. It potentially does great benefits to the early detection, staging, and treatment response of the diseases. As the personal information is involved in the clinical procedures, it comes closer to realize the personalized medicine. The development strongly depends on the conjunction between various fields, including diagnostic imaging, nanotechnologies, and -omics database analysis.

\section{Conflict of Interests}

The authors declare that there is no conflict of interests regarding the publication of this paper.

\section{References}

[1] N. G. Costouros, D. Lorang, Y. Zhang et al., "Microarray gene expression analysis of murine tumor heterogeneity defined by dynamic contrast-enhanced MRI," Molecular Imaging, vol. 1, no. 3, pp. 301-308, 2002.

[2] S. Guccione, Y.-S. Yang, G. Shi, D. Y. Lee, K. C. P. Li, and M. D. Bednarski, "Functional genomics guided with MR imaging: mouse tumor model study," Radiology, vol. 228, no. 2, pp. 560568, 2003.

[3] Y.-S. Yang, S. Guccione, and M. D. Bednarski, "Comparing genomic and histologic correlations to radiographic changes in tumors: a murine SCC VII model study," Academic Radiology, vol. 10, no. 10, pp. 1165-1175, 2003.

[4] S. K. Hobbs, G. Shi, R. Homer, G. Harsh, S. W. Atlas, and M. D. Bednarski, "Magnetic resonance image-guided proteomics of human glioblastoma multiforme," Journal of Magnetic Resonance Imaging, vol. 18, no. 5, pp. 530-536, 2003.

[5] M. D. Kuo, J. Gollub, C. B. Sirlin, C. Ooi, and X. Chen, "Radiogenomic analysis to identify imaging phenotypes associated with drug response gene expression programs in hepatocellular carcinoma," Journal of Vascular and Interventional Radiology, vol. 18, no. 7, pp. 821-830, 2007.

[6] E. Segal, C. B. Sirlin, C. Ooi et al., "Decoding global gene expression programs in liver cancer by noninvasive imaging," Nature Biotechnology, vol. 25, no. 6, pp. 675-680, 2007.

[7] M. Diehn, C. Nardini, D. S. Wang et al., "Identification of noninvasive imaging surrogates for brain tumor gene-expression modules," Proceedings of the National Academy of Sciences of the United States of America, vol. 105, no. 13, pp. 5213-5218, 2008.

[8] E. Segal, M. Shapira, A. Regev et al., "Module networks: identifying regulatory modules and their condition-specific regulators from gene expression data," Nature Genetics, vol. 34, no. 2, pp. 166-176, 2003.

[9] N. J. Serkova and M. S. Brown, "Quantitative analysis in magnetic resonance spectroscopy: from metabolic profiling to in vivo biomarkers," Bioanalysis, vol. 4, no. 3, pp. 321-341, 2012.

[10] S. Tiziani, Y. Kang, R. Harjanto et al., "Metabolomics of the tumor microenvironment in pediatric acute lymphoblastic leukemia," PLoS ONE, vol. 8, no. 12, Article ID e82859, 2013.

[11] G. Hassan-Smith, G. R. Wallace, M. R. Douglas, and A. J. Sinclair, "The role of metabolomics in neurological disease," Journal of Neuroimmunology, vol. 248, no. 1-2, pp. 48-52, 2012.

[12] E.-M. Spur, E. A. Decelle, and L. L. Cheng, "Metabolomic imaging of prostate cancer with magnetic resonance spectroscopy 
and mass spectrometry," European Journal of Nuclear Medicine and Molecular Imaging, vol. 40, supplement 1, pp. 60-71, 2013.

[13] R. Popovtzer, A. Agrawal, N. A. Kotov et al., "Targeted gold nanoparticles enable molecular CT imaging of cancer," Nano Letters, vol. 8, no. 12, pp. 4593-4596, 2008.

[14] T. Reuveni, M. Motiei, Z. Romman, A. Popovtzer, and R. Popovtzer, "Targeted gold nanoparticles enable molecular CT imaging of cancer: an in vivo study," International Journal of Nanomedicine, vol. 6, pp. 2859-2864, 2011.

[15] O. Rabin, J. M. Perez, J. Grimm, G. Wojtkiewicz, and R. Weissleder, "An X-ray computed tomography imaging agent based on long-circulating bismuth sulphide nanoparticles," Nature Materials, vol. 5, no. 2, pp. 118-122, 2006.

[16] B. Cohen, K. Ziv, V. Plaks et al., "MRI detection of transcriptional regulation of gene expression in transgenic mice," Nature Medicine, vol. 13, no. 4, pp. 498-503, 2007.

[17] A. A. Gilad, M. T. McMahon, P. Walczak et al., "Artificial reporter gene providing MRI contrast based on proton exchange," Nature Biotechnology, vol. 25, no. 2, pp. 217-219, 2007.

[18] C. H. Liu, S. Huang, J. Cui et al., "MR contrast probes that trace gene transcripts for cerebral ischemia in live animals," FASEB Journal, vol. 21, no. 11, pp. 3004-3015, 2007.

[19] A. Y. Louie, M. M. Hüber, E. T. Ahrens et al., "In vivo visualization of gene expression using magnetic resonance imaging," Nature Biotechnology, vol. 18, no. 3, pp. 321-325, 2000.

[20] J. B. Weaver, "Simultaneous multislice acquisition of MR images," Magnetic Resonance in Medicine, vol. 8, no. 3, pp. 275$284,1988$.

[21] F. A. Breuer, M. Blaimer, M. F. Mueller et al., "Controlled aliasing in volumetric parallel imaging (2D CAIPIRINHA)," Magnetic Resonance in Medicine, vol. 55, no. 3, pp. 549-556, 2006.

[22] S. A. Graham, D. J. Moseley, J. H. Siewerdsen, and D. A. Jaffray, "Compensators for dose and scatter management in cone-beam computed tomography," Medical Physics, vol. 34, no. 7, pp. 26912703, 2007.

[23] T. L. Toth, "Dose reduction opportunities for CT scanners," Pediatric Radiology, vol. 32, no. 4, pp. 261-267, 2002.

[24] C. H. McCollough, A. N. Primak, O. Saba et al., "Dose performance of a 64-channel dual-source CT scanner," Radiology, vol. 243, no. 3, pp. 775-784, 2007.

[25] S. S. Hsieh and N. J. Pelc, "The feasibility of a piecewise-linear dynamic bowtie filter," Medical Physics, vol. 40, no. 3, Article ID 031910, 2013.

[26] T. P. Szczykutowicz and C. A. Mistretta, "Design of a digital beam attenuation system for computed tomography: part I. System design and simulation framework," Medical Physics, vol. 40, no. 2, Article ID 021905, 2013.

[27] K. Perisinakis, A. E. Papadakis, and J. Damilakis, "The effect of X-ray beam quality and geometry on radiation utilization efficiency in multidetector CT imaging," Medical Physics, vol. 36, no. 4, pp. 1258-1266, 2009.

[28] A. Tzedakis, J. Damilakis, K. Perisinakis, J. Stratakis, and N. Gourtsoyiannis, "The effect of $\mathrm{z}$ overscanning on patient effective dose from multidetector helical computed tomography examinations," Medical Physics, vol. 32, no. 6, pp. 1621-1629, 2005.

[29] A. Tzedakis, J. Damilakis, K. Perisinakis, A. Karantanas, S. Karabekios, and N. Gourtsoyiannis, "Influence of z overscanning on normalized effective doses calculated for pediatric patients undergoing multidetector CT examinations," Medical Physics, vol. 34, no. 4, pp. 1163-1175, 2007.
[30] J. A. Christner, V. A. Zavaletta, C. D. Eusemann, A. I. WalzFlannigan, and C. H. McCollough, "Dose reduction in helical CT: dynamically adjustable $\mathrm{z}$-axis X-ray beam collimation," American Journal of Roentgenology, vol. 194, no. 1, pp. W49W55, 2010.

[31] P. D. Deak, O. Langner, M. Lell, and W. A. Kalender, "Effects of adaptive section collimation on patient radiation dose in multisection spiral CT,' Radiology, vol. 252, no. 1, pp. 140-147, 2009.

[32] P. M. Shikhaliev, T. Xu, and S. Molloi, "Photon counting computed tomography: concept and initial results," Medical Physics, vol. 32, no. 2, pp. 427-436, 2005.

[33] X. Wang, A. Zamyatin, and D. Shi, "Dose reduction potential with photon counting computed tomography," in Medical Imaging: Physics of Medical Imaging, N. J. Pelc, R. M. Nishikawa, and B. R. Whiting, Eds., vol. 8313 of Proceedings of SPIE, International Society for Optics and Photonics, February 2012.

[34] L. F. N. D. Carramate, F. Nachtrab, M. Firsching et al., "Energy resolving CT systems using Medipix2 and MHSP detectors," Journal of Instrumentation, vol. 8, no. 03, Article ID C03022, 2013.

[35] J. Giersch, D. Niederlöhner, and G. Anton, "The influence of energy weighting on X-ray imaging quality," Nuclear Instruments and Methods in Physics Research A, vol. 531, no. 1-2, pp. 68-74, 2004.

[36] K. S. Kalluri, M. Mahd, and S. J. Glick, "Investigation of energy weighting using an energy discriminating photon counting detector for breast CT," Medical Physics, vol. 40, no. 8, Article ID 081923, 2013.

[37] L. Wielopolski and R. P. Gardner, "Prediction of the pulseheight spectral distortion caused by the peak pile-up effect," Nuclear Instruments and Methods, vol. 133, no. 2, pp. 303-309, 1976.

[38] X. Wang, D. Meier, S. Mikkelsen et al., "MicroCT with energyresolved photon-counting detectors," Physics in Medicine and Biology, vol. 56, no. 9, pp. 2791-2816, 2011.

[39] A. Mohammadi, M. Baba, M. Nakhostin, H. Ohuchi, and M. Abe, "Compton spectroscopy for rotation-mode computed tomography," Journal of X-Ray Science and Technology, vol. 20, no. 2, pp. 131-140, 2012.

[40] J. Bennett, A. Opie, Q. Xu et al., "Hybrid spectral micro-CT: system design, implementation \& preliminary results," IEEE Transactions on Bio-Medical Engineering, vol. 61, no. 2, pp. 246253, 2014.

[41] W. A. Kalender, H. Wolf, and C. Suess, "Dose reduction in CT by anatomically adapted tube current modulation. II. Phantom measurements," Medical Physics, vol. 26, no. 11, pp. 2248-2253, 1999.

[42] J. R. Haaga, F. Miraldi, and W. MacIntyre, "The effect of mAs variation upon computed tomography image quality as evaluated by in vivo and in vitro studies," Radiology, vol. 138, no. 2, pp. 449-454, 1981.

[43] M. K. Kalra, M. M. Maher, T. L. Toth et al., "Techniques and applications of automatic tube current modulation for CT," Radiology, vol. 233, no. 3, pp. 649-657, 2004.

[44] H. Greeß, H. Wolf, U. Baum, W. A. Kalender, and W. Bautz, "Dose reduction in computed tomography by means of modulation of tube current according to anothing: first clinical results," RoFo, vol. 170, no. 3, pp. 246-250, 1999. 
[45] M. Sderberg and M. Gunnarsson, "Automatic exposure control in computed tomography an evaluation of systems from different manufacturers," Acta Radiologica, vol. 51, no. 6, pp. 625-634, 2010.

[46] W. Huda, E. M. Scalzetti, and G. Levin, "Technique factors and image quality as functions of patient weight at abdominal CT," Radiology, vol. 217, no. 2, pp. 430-435, 2000.

[47] B. B. Ertl-Wagner, R.-T. Hoffmann, R. Bruning et al., "Multidetector row $\mathrm{CT}$ angiography of the brain at various kilovoltage settings," Radiology, vol. 231, no. 2, pp. 528-535, 2004.

[48] Y. Funama, K. Awai, Y. Nakayama et al., "Radiation dose reduction without degradation of low-contrast detectability at abdominal multisection CT with a low-tube voltage technique: phantom study," Radiology, vol. 237, no. 3, pp. 905-910, 2005.

[49] B. Wintersperger, T. Jakobs, P. Herzog et al., "Aorto-iliac multidetector-row CT angiography with low $\mathrm{kV}$ settings: improved vessel enhancement and simultaneous reduction of radiation dose," European Radiology, vol. 15, no. 2, pp. 334-341, 2005.

[50] C. Schueller-Weidekamm, C. M. Schaefer-Prokop, M. Weber, C. J. Herold, and M. Prokop, "CT angiography of pulmonary arteries to detect pulmonary embolism: improvement of vascular enhancement with low kilovoltage settings," Radiology, vol. 241, no. 3, pp. 899-907, 2006.

[51] A. Waaijer, M. Prokop, B. K. Velthuis, C. J. G. Bakker, G. A. P. De Kort, and M. S. Van Leeuwen, "Circle of Willis at CT angiography: dose reduction and image quality_reducing tube voltage and increasing tube current settings," Radiology, vol. 242, no. 3, pp. 832-839, 2007.

[52] Y. Nakayama, K. Awai, Y. Funama et al., "Abdominal CT with low tube voltage: preliminary observations about radiation dose, contrast enhancement, image quality, and noise," Radiology, vol. 237, no. 3, pp. 945-951, 2005.

[53] J. Nuyts, B. De Man, P. Dupont, M. Defrise, P. Suetens, and L. Mortelmans, "Iterative reconstruction for helical CT: a simulation study," Physics in Medicine and Biology, vol. 43, no. 4, pp. 729-737, 1998.

[54] J.-B. Thibault, K. D. Sauer, C. A. Bouman, and J. Hsieh, "A threedimensional statistical approach to improved image quality for multislice helical CT,' Medical Physics, vol. 34, no. 11, pp. 45264544, 2007.

[55] I. A. Elbakri and J. A. Fessler, "Statistical image reconstruction for polyenergetic X-ray computed tomography," IEEE Transactions on Medical Imaging, vol. 21, no. 2, pp. 89-99, 2002.

[56] A. K. Hara, R. G. Paden, A. C. Silva, J. L. Kujak, H. J. Lawder, and W. Pavlicek, "Iterative reconstruction technique for reducing body radiation dose at CT: feasibility study," American Journal of Roentgenology, vol. 193, no. 3, pp. 764-771, 2009.

[57] J. S. Kole and F. J. Beekman, "Evaluation of accelerated iterative X-ray CT image reconstruction using floating point graphics hardware," Physics in Medicine and Biology, vol. 51, no. 4, pp. 875-889, 2006.

[58] F. Xu and K. Mueller, "Real-time 3D computed tomographic reconstruction using commodity graphics hardware," Physics in Medicine and Biology, vol. 52, no. 12, article 006, pp. 3405-3419, 2007.

[59] G. C. Sharp, N. Kandasamy, H. Singh, and M. Folkert, "GPUbased streaming architectures for fast cone-beam CT image reconstruction and demons deformable registration," Physics in Medicine and Biology, vol. 52, no. 19, article 003, pp. 5771-5783, 2007.
[60] H. Kudo, T. Suzuki, and E. A. Rashed, "Image reconstruction for sparse-view CT and interior CT-introduction to compressed sensing and differentiated backprojection," Quantitative Imaging in Medicine and Surgery, vol. 3, no. 3, pp. 147-161, 2013.

[61] E. J. Candès, J. Romberg, and T. Tao, "Robust uncertainty principles: exact signal reconstruction from highly incomplete frequency information," IEEE Transactions on Information Theory, vol. 52, no. 2, pp. 489-509, 2006.

[62] D. L. Donoho, “Compressed sensing," IEEE Transactions on Information Theory, vol. 52, no. 4, pp. 1289-1306, 2006.

[63] G.-H. Chen, J. Tang, and S. Leng, "Prior Image Constrained Compressed Sensing (PICCS): a method to accurately reconstruct dynamic CT images from highly undersampled projection data sets," Medical Physics, vol. 35, no. 2, pp. 660-663, 2008.

[64] E. Y. Sidky and X. Pan, "Image reconstruction in circular conebeam computed tomography by constrained, total-variation minimization," Physics in Medicine and Biology, vol. 53, no. 17, pp. 4777-4807, 2008.

[65] K. Choi, J. Wang, L. Zhu, T.-S. Suh, S. Boyd, and L. Xing, "Compressed sensing based cone-beam computed tomography reconstruction with a first-order method," Medical Physics, vol. 37, no. 9, pp. 5113-5125, 2010.

[66] L. Ritschl, F. Bergner, C. Fleischmann, and M. Kachelriess, "Improved total variation-based CT image reconstruction applied to clinical data," Physics in Medicine and Biology, vol. 56, no. 6, pp. 1545-1561, 2011.

[67] E. Y. Sidky, C.-M. Kao, and X. Pan, "Accurate image reconstruction from few-views and limited-angle data in divergent-beam CT,' Journal of X-Ray Science and Technology, vol. 14, no. 2, pp. 119-139, 2006.

[68] F. Noo, R. Clackdoyle, and J. D. Pack, "A two-step Hilbert transform method for $2 \mathrm{D}$ image reconstruction," Physics in Medicine and Biology, vol. 49, no. 17, pp. 3903-3923, 2004.

[69] X. Pan, Y. Zou, and D. Xia, "Image reconstruction in peripheral and central regions-of-interest and data redundancy," Medical Physics, vol. 32, no. 3, pp. 673-684, 2005.

[70] K. Ogawa, M. Nakajima, and S. Yuta, "A reconstruction algorithm from truncated projections," IEEE Transactions on Medical Imaging, vol. 3, no. 1, pp. 34-40, 1984.

[71] B. Ohnesorge, T. Flohr, K. Schwarz, J. P. Heiken, and K. T. Bae, "Efficient correction for CT image artifacts caused by objects extending outside the scan field of view," Medical Physics, vol. 27, no. 1, pp. 39-46, 2000.

[72] S. Kappler and S. Wirth, "Comparison Of dual-kVp and duallayer CT in simulations and real CT system measurements," in Proceedings of the IEEE Nuclear Science Symposium Conference Record (NSS/MIC '08), pp. 4828-4831, IEEE, October 2008.

[73] L. Goshen, J. Sosna, R. Carmi, G. Kafri, I. Iancu, and A. Altman, "An iodine-calcium separation analysis and virtually non-contrasted image generation obtained with single source dual energy MDCT," in Proceedings of the IEEE Nuclear Science Symposium Conference Record (NSS/MIC '08), pp. 3868-3870, IEEE, October 2008.

[74] Y. Zou and M. D. Silver, "Analysis of fast $\mathrm{kV}$-switching in dual energy CT using a pre-reconstruction decomposition technique," in Medical Imaging: Physics of Medical Imaging, vol. 6913 of Proceedings of SPIE, International Society for Optics and Photonics, February 2008.

[75] T. R. C. Johnson, B. Krauss, M. Sedlmair et al., "Material differentiation by dual energy CT: initial experience," European Radiology, vol. 17, no. 6, pp. 1510-1517, 2007. 
[76] T. G. Flohr, H. Bruder, K. Stierstorfer, M. Petersilka, B. Schmidt, and C. H. McCollough, "Image reconstruction and image quality evaluation for a dual source CT scanner," Medical Physics, vol. 35, no. 12, pp. 5882-5897, 2008.

[77] A. N. Primak, J. C. R. Giraldo, X. Liu, L. Yu, and C. H. McCollough, "Improved dual-energy material discrimination for dual-source CT by means of additional spectral filtration," Medical Physics, vol. 36, no. 4, pp. 1359-1369, 2009.

[78] M. Karçaaltıncaba and A. Aktaş, "Dual-energy CT revisited with multidetector CT: review of principles and clinical applications," Diagnostic and Interventional Radiology, vol. 17, no. 3, pp. 181-194, 2011.

[79] T. Kraśnicki, P. Podgórski, M. Guziński et al., "Novel clinical applications of dual energy computed tomography," Advances in Clinical and Experimental Medicine, vol. 21, no. 6, pp. 831-841, 2012.

[80] T. Heye, R. C. Nelson, L. M. Ho, D. Marin, and D. T. Boll, "Dualenergy CT applications in the abdomen," American Journal of Roentgenology, vol. 199, supplement 5, pp. 64-70, 2012.

[81] H. Scheffel, P. Stolzmann, T. Frauenfelder et al., "Dual-energy contrast-enhanced computed tomography for the detection of urinary stone disease," Investigative Radiology, vol. 42, no. 12, pp. 823-829, 2007.

[82] N. Takahashi, R. P. Hartman, T. J. Vrtiska et al., "Dual-energy CT iodine-subtraction virtual unenhanced technique to detect urinary stones in an iodine-filled collecting system: a phantom study," American Journal of Roentgenology, vol. 190, no. 5, pp. 1169-1173, 2008.

[83] G. Ascenti, A. Mileto, M. Gaeta, A. Blandino, S. Mazziotti, and E. Scribano, "Single-phase dual-energy CT urography in the evaluation of haematuria," Clinical Radiology, vol. 68, no. 2, pp. 87-94, 2013.

[84] A. Graser, T. R. C. Johnson, E. M. Hecht et al., "Dual-energy CT in patients suspected of having renal masses: can virtual nonenhanced images replace true nonenhanced images?" Radiology, vol. 252, no. 2, pp. 433-440, 2009.

[85] A. Graser, C. R. Becker, M. Staehler et al., "Single-phase dualenergy CT allows for characterization of renal masses as benign or malignant," Investigative Radiology, vol. 45 , no. 7, pp. 399405, 2010.

[86] S. H. Lee, J. Hur, Y. J. Kim, H.-J. Lee, Y. J. Hong, and B. W. Choi, "Additional value of dual-energy CT to differentiate between benign and malignant mediastinal tumors: an initial experience," European Journal of Radiology, vol. 82, no. 11, pp. 2043-2049, 2013.

[87] G. Schmid-Bindert, T. Henzler, T. Q. Chu et al., "Functional imaging of lung cancer using dual energy CT: how does iodine related attenuation correlate with standardized uptake value of 18FDG-PET-CT?" European Radiology, vol. 22, no. 1, pp. 93-103, 2012.

[88] M. Meyer, P. Hohenberger, P. Apfaltrer et al., "CT-based response assessment of advanced gastrointestinal stromal tumor: dual energy CT provides a more predictive imaging biomarker of clinical benefit than RECIST or Choi criteria," European Journal of Radiology, vol. 82, no. 6, pp. 923-928, 2013.

[89] K. A. Miles and M. R. Griffiths, "Perfusion CT: a worthwhile enhancement?" British Journal of Radiology, vol. 76, no. 904, pp. 220-231, 2003.

[90] A. R. Kambadakone and D. V. Sahani, "Body perfusion CT: technique, clinical applications, and advances," Radiologic Clinics of North America, vol. 47, no. 1, pp. 161-178, 2009.
[91] C.-J. Sun, C. Li, H.-B. Lv et al., "Comparing CT perfusion with oxygen partial pressure in a rabbit VX2 soft-tissue tumor model," Journal of Radiation Research, vol. 55, no. 1, pp. 183-190, 2014.

[92] G.-L. Ma, R.-J. Bai, H.-J. Jiang et al., "Early changes of hepatic hemodynamics measured by functional CT perfusion in a rabbit model of liver tumor," Hepatobiliary \& Pancreatic Diseases International, vol. 11, no. 4, pp. 407-411, 2012.

[93] R. Jain, "Perfusion CT imaging of brain tumors: an overview," American Journal of Neuroradiology, vol. 32, no. 9, pp. 1570-1577, 2011.

[94] D. Arandjic, F. Bonutti, E. Biasizzo et al., "Radiation doses in cerebral perfusion computed tomography: patient and phantom study," Radiation Protection Dosimetry, vol. 154, no. 4, pp. 459464, 2013.

[95] N. Negi, T. Yoshikawa, Y. Ohno et al., "Hepatic CT perfusion measurements: a feasibility study for radiation dose reduction using new image reconstruction method," European Journal of Radiology, vol. 81, no. 11, pp. 3048-3054, 2012.

[96] R. Krissak, C. A. Mistretta, T. Henzler et al., "Noise reduction and image quality improvement of low dose and ultra low dose brain perfusion CT by HYPR-LR processing," PLOS ONE, vol. 6, no. 2, Article ID e17098, 2011.

[97] M. Supanich, Y. Tao, B. Nett et al., "Radiation dose reduction in time-resolved CT angiography using highly constrained back projection reconstruction," Physics in Medicine and Biology, vol. 54, no. 14, pp. 4575-4593, 2009.

[98] L. He, B. Orten, S. Do et al., "A spatio-temporal deconvolution method to improve perfusion CT quantification," IEEE Transactions on Medical Imaging, vol. 29, no. 5, pp. 1182-1191, 2010.

[99] H. Yu, S. Zhao, E. A. Hoffman, and G. Wang, "Ultra-low dose lung CT perfusion regularized by a previous scan," Academic Radiology, vol. 16, no. 3, pp. 363-373, 2009.

[100] J. J. S. Shankar, C. Lum, and M. Sharma, "Whole-brain perfusion imaging with 320-MDCT scanner: reducing radiation dose by increasing sampling interval," American Journal of Roentgenology, vol. 195, no. 5, pp. 1183-1186, 2010.

[101] A. Tognolini, R. Schor-Bardach, O. S. Pianykh, C. J. Wilcox, V. Raptopoulos, and S. N. Goldberg, "Body tumor CT perfusion protocols: optimization of acquisition scan parameters in a rat tumor model," Radiology, vol. 251, no. 3, pp. 712-720, 2009.

[102] P. C. Lauterbur, "Image formation by induced local interactions: examples employing nuclear magnetic resonance," Nature, vol. 242, no. 5394, pp. 190-191, 1973.

[103] H. Pedersen, S. Kozerke, S. Ringgaard, K. Nehrke, and Y. K. Won, "K-t PCA: temporally constrained k-t BLAST reconstruction using principal component analysis," Magnetic Resonance in Medicine, vol. 62, no. 3, pp. 706-716, 2009.

[104] L. Ge, A. Kino, M. Griswold, C. Mistretta, J. C. Carr, and D. $\mathrm{Li}$, "Myocardial perfusion MRI with sliding-window conjugategradient HYPR," Magnetic Resonance in Medicine, vol. 62, no. 4, pp. 835-839, 2009.

[105] B. Jung, M. Honal, J. Hennig, and M. Markl, "k-t-space accelerated myocardial perfusion," Journal of Magnetic Resonance Imaging, vol. 28, no. 5, pp. 1080-1085, 2008.

[106] P. Kellman, J. A. Derbyshire, K. O. Agyeman, E. R. McVeigh, and A. E. Arai, "Extended coverage first-pass perfusion imaging using slice-interleaved TSENSE," Magnetic Resonance in Medicine, vol. 51, no. 1, pp. 200-204, 2004.

[107] J.-S. Hsu, S.-Y. Tsai, M.-T. Wu, H.-W. Chung, and Y.-R. Lin, "Fast dynamic contrast-enhanced lung MR imaging using $\mathrm{k}-\mathrm{t}$ BLAST: 
a spatiotemporal perspective," Magnetic Resonance in Medicine, vol. 67, no. 3, pp. 786-792, 2012.

[108] C. A. Mistretta, O. Wieben, J. Velikina et al., "Highly constrained backprojection for time-resolved MRI," Magnetic Resonance in Medicine, vol. 55, no. 1, pp. 30-40, 2006.

[109] Y. Wu, N. Kim, F. R. Korosec et al., "3D time-resolved contrastenhanced cerebrovascular MR angiography with subsecond frame update times using radial k-space trajectories and highly constrained projection reconstruction," American Journal of Neuroradiology, vol. 28, no. 10, pp. 2001-2004, 2007.

[110] K. K. Vigen, D. C. Peters, T. M. Grist, W. F. Block, and C. A. Mistretta, "Undersampled projection-reconstruction imaging for time-resolved contrast-enhanced imaging," Magnetic Resonance in Medicine, vol. 43, no. 2, pp. 170-176, 2000.

[111] F. R. Korosec, R. Frayne, T. M. Grist, and C. A. Mistretta, “Timeresolved contrast-enhanced 3D MR angiography," Magnetic Resonance in Medicine, vol. 36, no. 3, pp. 345-351, 1996.

[112] J. F. Utting, S. Kozerke, R. Schnitker, and T. Niendorf, "Comparison of k-t SENSE/k-t BLAST with conventional SENSE applied to BOLD fMRI," Journal of Magnetic Resonance Imaging, vol. 32, no. 1, pp. 235-241, 2010.

[113] B. Madore, G. H. Glover, and N. J. Pelc, "Unaliasing by fourier-encoding the overlaps using the temporal dimension (UNFOLD), applied to cardiac imaging and fMRI," Magnetic Resonance in Medicine, vol. 42, no. 5, pp. 813-828, 1999.

[114] D. C. Peters, D. B. Ennis, and E. R. McVeigh, "High-resolution MRI of cardiac function with projection reconstruction and steady-state free precession," Magnetic Resonance in Medicine, vol. 48, no. 1, pp. 82-88, 2002.

[115] B. Jung, P. Ullmann, M. Honal, S. Bauer, J. Hennig, and M. Markl, "Parallel MRI with extended and averaged GRAPPA kernels (PEAK-GRAPPA): optimized spatiotemporal dynamic imaging," Journal of Magnetic Resonance Imaging, vol. 28, no. 5, pp. 1226-1232, 2008.

[116] P. Kellman, F. H. Epstein, and E. R. McVeigh, "Adaptive sensitivity encoding incorporating temporal filtering (TSENSE)," Magnetic Resonance in Medicine, vol. 45, no. 5, pp. 846-852, 2001.

[117] J. Tsao, S. Kozerke, P. Boesiger, and K. P. Pruessmann, "Optimizing spatiotemporal sampling for $k-t$ BLAST and k-t SENSE: application to high-resolution real-time cardiac steady-state free precession," Magnetic Resonance in Medicine, vol. 53, no. 6, pp. 1372-1382, 2005.

[118] M. A. Griswold, P. M. Jakob, R. M. Heidemann et al., "Generalized Autocalibrating Partially Parallel Acquisitions (GRAPPA)," Magnetic Resonance in Medicine, vol. 47, no. 6, pp. 1202-1210, 2002.

[119] D. K. Sodickson and W. J. Manning, "Simultaneous Acquisition of Spatial Harmonics (SMASH): fast imaging with radiofrequency coil arrays," Magnetic Resonance in Medicine, vol. 38, no. 4, pp. 591-603, 1997.

[120] P. M. Jakob, M. A. Griswold, R. R. Edelman, and D. K. Sodickson, "AUTO-SMASH: a self-calibrating technique for SMASH imaging," Magnetic Resonance Materials in Physics, Biology and Medicine, vol. 7, no. 1, pp. 42-54, 1998.

[121] R. Heidemann and M. Griswold, "VD-AUTO-SMASH imaging," Magnetic Resonance in Medicine, vol. 45, no. 6, pp. 10661074, 2000.

[122] P. J. Beatty, A. C. Brau, and S. Chang, "A method for autocalibrating 2-D accelerated volumetric parallel imaging with clinically practical reconstruction times," in Proceedings of the
International Society for Magnetic Resonance (ISMRM '07), vol. 15, p. 1749, Berlin, Germany, 2007.

[123] M. Blaimer, F. A. Breuer, M. Mueller et al., "2D-GRAPPAoperator for faster 3D parallel MRI," Magnetic Resonance in Medicine, vol. 56, no. 6, pp. 1359-1364, 2006.

[124] K. Pruessmann, M. Weiger, M. Scheidegger, and P. Boesiger, "SENSE: sensitivity encoding for fast MRI," Magnetic Resonance in Medicine, vol. 42, pp. 952-962, 1999.

[125] K. Ocegueda and A. O. Rodriguez, "Slotted surface coil with reduced g-factor for SENSE imaging," in Proceedings of the 28th Annual International Conference of the IEEE Engineering in Medicine and Biology Society (EMBS '06), vol. 1, pp. 1904-1906, September 2006.

[126] L. T. Muftuler, G. Chen, and O. Nalcioglu, "An inverse method to design RF coil arrays optimized for SENSE imaging," Physics in Medicine and Biology, vol. 51, no. 24, article 012, pp. 64576469, 2006.

[127] B. Liu, K. King, M. Steckner, J. Xie, J. Sheng, and L. Ying, "Regularized sensitivity encoding (SENSE) reconstruction using bregman iterations," Magnetic Resonance in Medicine, vol. 61, no. 1, pp. 145-152, 2009.

[128] D. Liang, H. Wang, and L. Ying, "SENSE reconstruction with nonlocal TV regularization," in Proceedings of the Annual International Conference of the IEEE Engineering in Medicine and Biology Society (EMBC '09), pp. 1032-1035, 2009.

[129] F.-H. Lin, K. K. Kwong, J. W. Belliveau, and L. L. Wald, "Parallel imaging reconstruction using automatic regularization," Magnetic Resonance in Medicine, vol. 51, no. 3, pp. 559-567, 2004.

[130] A. A. Samsonov, "On optimality of parallel MRI reconstruction in k-space," Magnetic Resonance in Medicine, vol. 59, no. 1, pp. 156-164, 2008.

[131] S. O. Schönberg, O. Dietrich, and M. F. Reiser, Parallel Imaging in Clinical MR Applications, Springer, 2007.

[132] M. N. J. Paley, K. J. Lee, J. M. Wild, P. D. Griffiths, and E. H. Whitby, "Simultaneous parallel inclined readout image technique," Magnetic Resonance Imaging, vol. 24, no. 5, pp. 557$562,2006$.

[133] F. A. Breuer, M. Blaimer, R. M. Heidemann, M. F. Mueller, M. A. Griswold, and P. M. Jakob, "Controlled aliasing in parallel imaging results in higher acceleration (CAIPIRINHA) for multi-slice imaging," Magnetic Resonance in Medicine, vol. 53, no. 3, pp. 684-691, 2005.

[134] D. Stäb, C. O. Ritter, F. A. Breuer, A. M. Weng, D. Hahn, and H. Köstler, "CAIPIRINHA accelerated SSFP imaging," Magnetic Resonance in Medicine, vol. 65, no. 1, pp. 157-164, 2011.

[135] S. R. Yutzy, N. Seiberlich, J. L. Duerk, and M. A. Griswold, "Improvements in multislice parallel imaging using radial CAIPIRINHA," Magnetic Resonance in Medicine, vol. 65, no. 6, pp. 1630-1637, 2011.

[136] H. J. Michaely, J. N. Morelli, J. Budjan et al., "CAIPIRINHADixon-TWIST (CDT)-volume-interpolated breath-hold examination (VIBE): a new technique for fast time-resolved dynamic 3-dimensional imaging of the abdomen with high spatial resolution," Investigative Radiology, vol. 48, no. 8, pp. 590-597, 2013.

[137] M. H. Yu, J. M. Lee, J.-H. Yoon, B. Kiefer, J. K. Han, and B.I. Choi, "Clinical application of controlled aliasing in parallel imaging results in a higher acceleration (CAIPIRINHA)volumetric interpolated breathhold (VIBE) sequence for gadoxetic acid-enhanced liver MR imaging," Journal of Magnetic Resonance Imaging, vol. 38, no. 5, pp. 1020-1026, 2013. 
[138] R. Nunes, J. Hajnal, X. Golay, and D. Larkman, "Simultaneous slice excitation and reconstruction for single shot EPI, in Proceedings of the International Society for Magnetic Resonance in Medicine (ISMRM '06), vol. 14, p. 293, 2006.

[139] K. Setsompop, B. A. Gagoski, J. R. Polimeni, T. Witzel, V. J. Wedeen, and L. L. Wald, "Blipped-controlled aliasing in parallel imaging for simultaneous multislice echo planar imaging with reduced g-factor penalty," Magnetic Resonance in Medicine, vol. 67, no. 5, pp. 1210-1224, 2012.

[140] S. J. Riederer, T. Tasciyan, F. Farzaneh, J. N. Lee, R. C. Wright, and R. J. Herfkens, "MR fluoroscopy: technical feasibility," Magnetic Resonance in Medicine, vol. 8, no. 1, pp. 1-15, 1988.

[141] J. J. van Vaals, M. E. Brummer, W. T. Dixon et al., "Keyhole" method for accelerating imaging of contrast agent uptake," Journal of Magnetic Resonance Imaging, vol. 3, no. 4, pp. 671675, 1993

[142] M. Doyle, E. G. Walsh, G. G. Blackwell, and G. M. Pohost, "Block regional interpolation scheme for $\mathrm{k}$-space (BRISK): a rapid cardiac imaging technique," Magnetic Resonance in Medicine, vol. 33, no. 2, pp. 163-170, 1995.

[143] T. Song, A. F. Laine, Q. Chen et al., "Optimal k-space sampling for dynamic contrast-enhanced MRI with an application to MR renography," Magnetic Resonance in Medicine, vol. 61, no. 5, pp. 1242-1248, 2009.

[144] R. P. Lim, M. Shapiro, E. Y. Wang et al., "3D time-resolved MR angiography (MRA) of the carotid arteries with timeresolved imaging with stochastic trajectories: comparison with 3D contrast-enhanced bolus-chase MRA and 3D time-of-flight MRA," American Journal of Neuroradiology, vol. 29, no. 10, pp. 1847-1854, 2008

[145] J. Tsao, P. Boesiger, and K. P. Pruessmann, "k-t BLAST and k-t SENSE: dynamic MRI with high frame rate exploiting spatiotemporal correlations," Magnetic Resonance in Medicine, vol. 50, no. 5, pp. 1031-1042, 2003.

[146] F. Huang, J. Akao, S. Vijayakumar, G. R. Duensing, and M. Limkeman, "K-t GRAPPA: a k-space implementation for dynamic MRI with high reduction factor," Magnetic Resonance in Medicine, vol. 54, no. 5, pp. 1172-1184, 2005.

[147] S. Schnell, M. Markl, P. Entezari et al., "k-t GRAPPA accelerated four-dimensional flow MRI in the aorta: effect on scan time, image quality, and quantification of flow and wall shear stress," Magnetic Resonance in Medicine, 2013.

[148] P. Lai, F. Huang, A. C. Larson, and D. Li, "Fast four-dimensional coronary MR angiography with k-t GRAPPA," Journal of Magnetic Resonance Imaging, vol. 27, no. 3, pp. 659-665, 2008.

[149] M. Lustig, D. Donoho, and J. M. Pauly, "Sparse MRI: the application of compressed sensing for rapid MR imaging," Magnetic Resonance in Medicine, vol. 58, no. 6, pp. 1182-1195, 2007.

[150] U. Gamper, P. Boesiger, and S. Kozerke, "Compressed sensing in dynamic MRI," Magnetic Resonance in Medicine, vol. 59, no. 2, pp. 365-373, 2008.

[151] M. S. Hansen, C. Baltes, J. Tsao, S. Kozerke, K. P. Pruessmann, and H. Eggers, "k-t BLAST reconstruction from non-Cartesian k-t space sampling," Magnetic Resonance in Medicine, vol. 55, no. 1, pp. 85-91, 2006.

[152] C. M. Tsai and D. G. Nishimura, "Reduced aliasing artifacts using variable-density k-space sampling trajectories," Magnetic Resonance in Medicine, vol. 43, no. 3, pp. 452-458, 2000.

[153] K. Scheffler and J. Hennig, "Reduced circular field-of-view imaging," Magnetic Resonance in Medicine, vol. 40, no. 3, pp. 474-480, 1998.
[154] M. Doneva, P. Börnert, H. Eggers, A. Mertins, J. Pauly, and M. Lustig, "Compressed sensing for chemical shift-based water-fat separation," Magnetic Resonance in Medicine, vol. 64, no. 6, pp. $1749-1759,2010$

[155] C. N. Wiens, C. M. McCurdy, J. D. Willig-Onwuachi, and C. A. McKenzie, "R2*-corrected water-fat imaging using compressed sensing and parallel imaging," Magnetic Resonance in Medicine, 2013.

[156] R. Otazo, D. Kim, L. Axel, and D. K. Sodickson, "Combination of compressed sensing and parallel imaging for highly accelerated first-pass cardiac perfusion MRI," Magnetic Resonance in Medicine, vol. 64, no. 3, pp. 767-776, 2010.

[157] D. Kim, H. A. Dyvorne, R. Otazo, L. Feng, D. K. Sodickson, and V. S. Lee, "Accelerated phase-contrast cine MRI using k-t SPARSE-SENSE," Magnetic Resonance in Medicine, vol. 67, no. 4, pp. 1054-1064, 2012.

[158] H. Jung, K. Sung, K. S. Nayak, E. Y. Kim, and J. C. Ye, "Kt FOCUSS: a general compressed sensing framework for high resolution dynamic MRI," Magnetic Resonance in Medicine, vol. 61, no. 1, pp. 103-116, 2009.

[159] M. Akçakaya, T. A. Basha, R. H. Chan, W. J. Manning, and R. Nezafat, "Accelerated isotropic sub-millimeter whole-heart coronary MRI: compressed sensing versus parallel imaging," Magnetic Resonance in Medicine, 2013.

[160] A. Hsiao, M. Lustig, M. T. Alley, M. J. Murphy, and S. S. Vasanawala, "Evaluation of valvular insufficiency and shunts with parallel-imaging compressed-sensing $4 \mathrm{D}$ phase-contrast MR imaging with stereoscopic 3D velocity-fusion volumerendered visualization," Radiology, vol. 265, no. 1, pp. 87-95, 2012.

[161] H. Nguyen and G. Glover, "A modified generalized series approach: application to sparsely sampled FMRI," IEEE Transactions on Biomedical Engineering, vol. 60, no. 10, pp. 2867-2877, 2013.

[162] D. J. Holland, C. Liu, X. Song et al., "Compressed sensing reconstruction improves sensitivity of variable density spiral fMRI," Magnetic Resonance in Medicine, vol. 70, no. 6, pp. 16341643, 2013. 


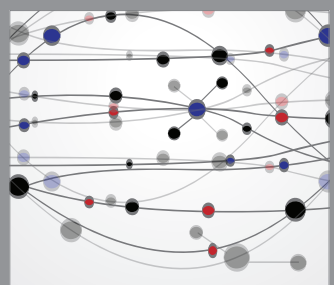

The Scientific World Journal
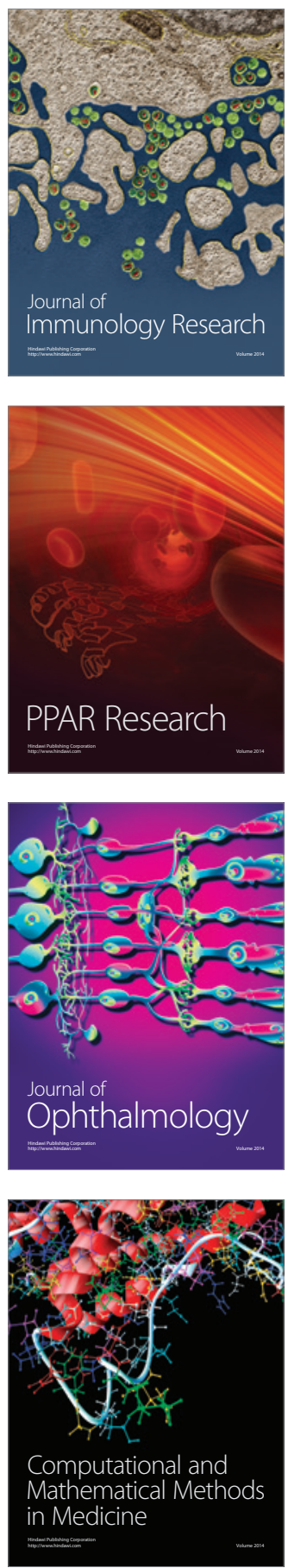

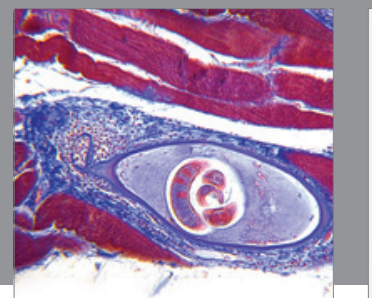

Gastroenterology

Research and Practice
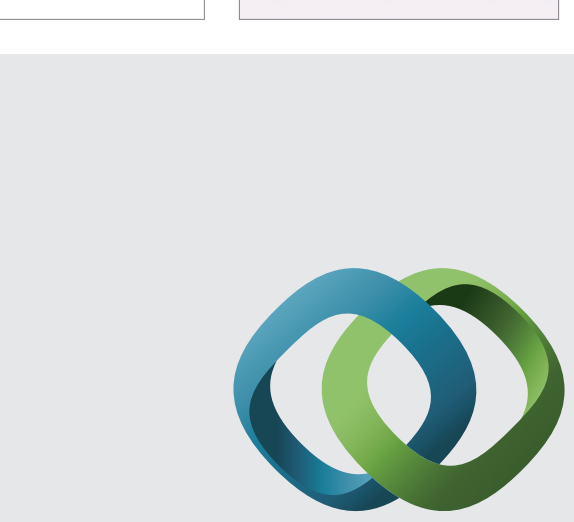

\section{Hindawi}

Submit your manuscripts at

http://www.hindawi.com
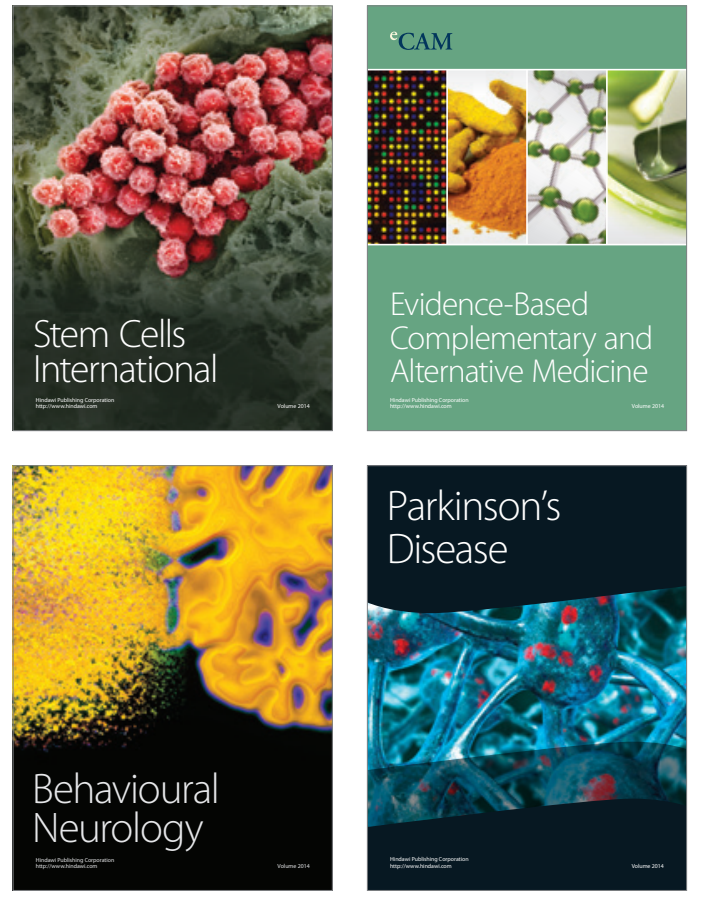
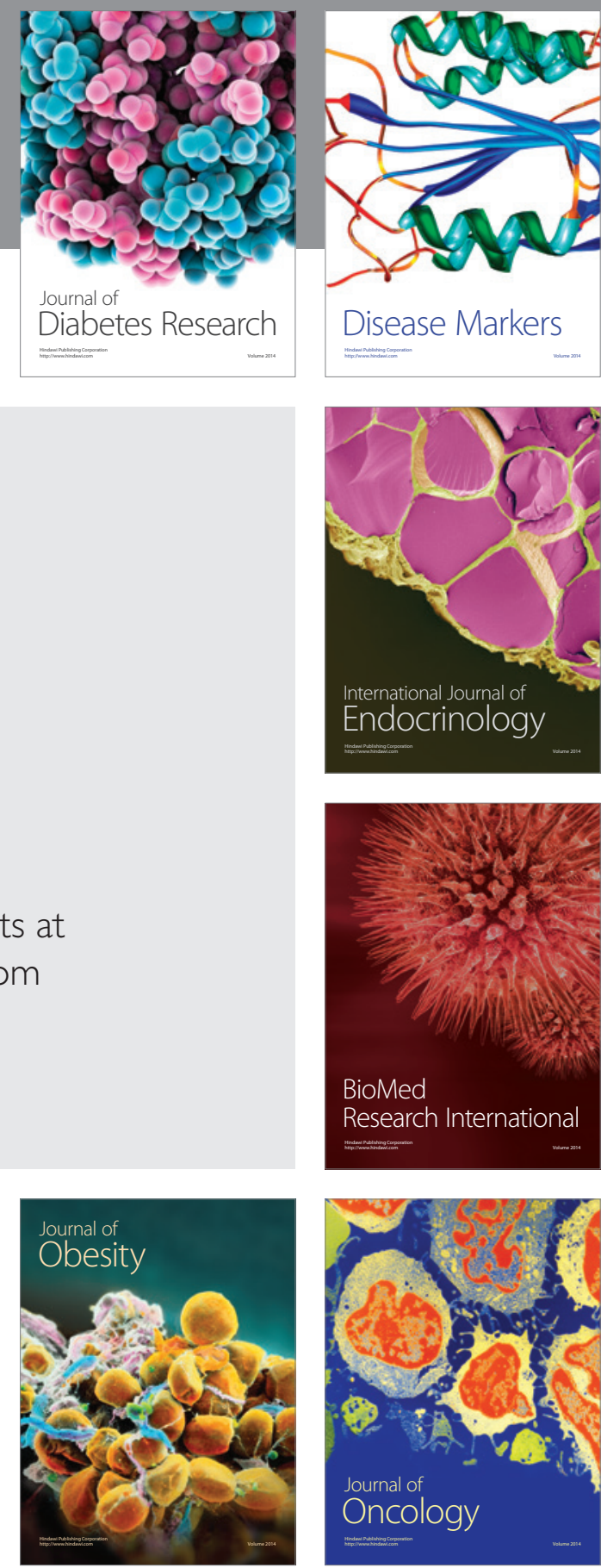

Disease Markers
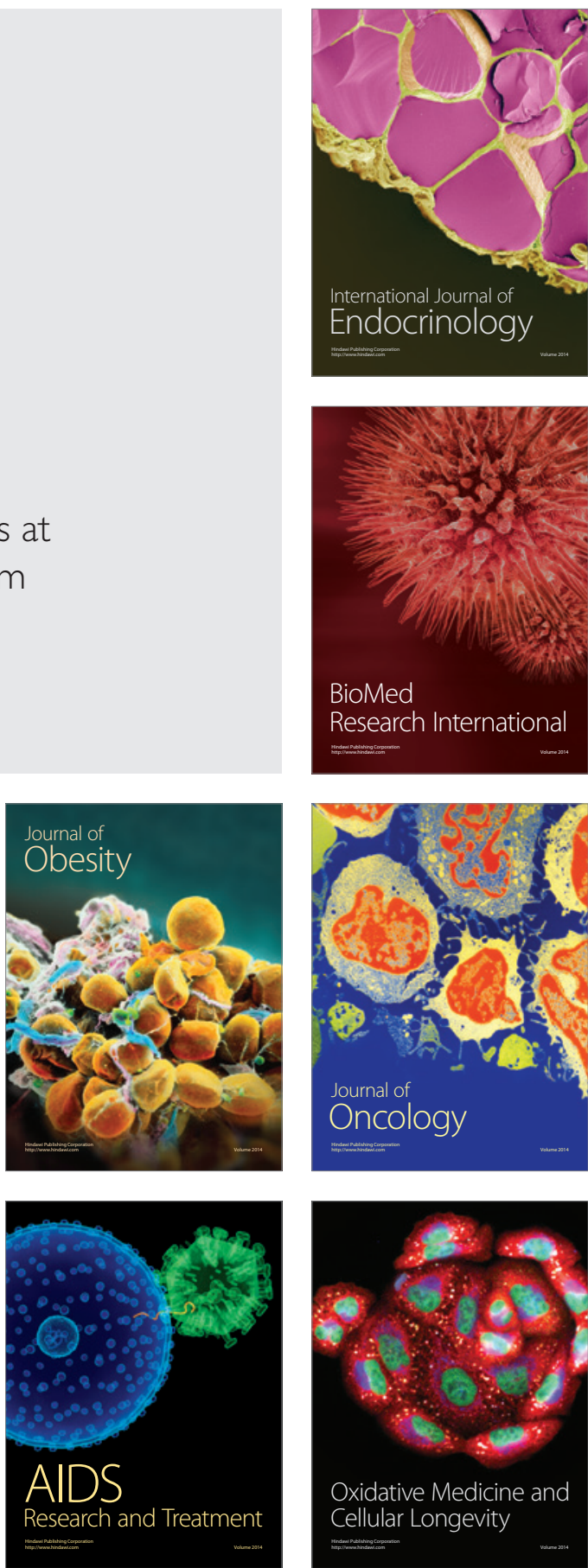